\title{
BISHOP AND LAPLACIAN COMPARISON THEOREMS ON SASAKIAN MANIFOLDS
}

\author{
PAUL W. Y. LEE AND CHENGBO LI
}

\begin{abstract}
We prove a Bishop volume comparison theorem and a Laplacian comparison theorem for a natural sub-Riemannian structure defined on Sasakian manifolds. This generalizes the earlier work in [6, 5, 1] for the three dimensional case.
\end{abstract}

\section{INTRODUCTION}

Bishop volume comparison theorem and Laplacian comparison theorem are basic tools in Riemannian geometry and geometric analysis. In this paper, we prove an analogue for a natural sub-Riemannian structure defined on a Sasakian manifold.

Recall that a Sasakian manifold is a $2 n+1$-dimensional manifold $M$ equipped with the an almost contact structure $\left(\mathbf{J}, \alpha_{0}, v_{0}\right)$ and a Riemannian metric $\langle\cdot, \cdot\rangle$ satisfying certain compatibility conditions (see Section 3 for the definitions). The restriction of the Riemannian metric on the distribution $\mathcal{D}:=\operatorname{ker} \alpha_{0}$ defines a sub-Riemannian structure. Let $B_{x}(R)$ be the sub-Riemannian ball of radius $R$ centered at $x$ and let $\eta$ be the Riemannian volume form of the Riemannian metric $\langle\cdot, \cdot\rangle$. The Heisenberg group and the complex Hopf fibration are well-known Sasakian manifolds (see Section 7 for more detail). Their volume forms are denoted, respectively, by $\eta_{0}$ and $\eta_{H}$. We also denote their subRiemannian balls by and $B_{0}(R)$ and $B_{H}(R)$, respectively. The following Bishop type volume comparison theorems generalize the earlier three dimensional case in [6, 5, 1].

Theorem 1.1. Assume that the Tanaka-Webster curvature Rm* of the Sasakian manifold satisfies

(1) $\left\langle R m^{*}(\boldsymbol{J} v, v) v, \boldsymbol{J} v\right\rangle \geq 0$,

(2) $\sum_{i=1}^{2 n-2}\left\langle R m^{*}\left(w_{i}, v\right) v, w_{i}\right\rangle \geq 0$,

Date: September 13, 2018.

The first author's research was supported by the Research Grant Council of Hong Kong (RGC Ref. No. CUHK404512). The second author was supported in part by the National Natural Science Foundation of China (Grant No. 11201330). 
where $v$ is any vector in $\mathcal{D}$ and $w_{1}, \ldots, w_{2 n-2}$ in an orthonormal frame of $\left\{v_{0}, v, \boldsymbol{J} v\right\}^{\perp}$. Then

$$
\eta\left(B_{x}(R)\right) \leq \eta_{0}\left(B_{0}(R)\right)
$$

Moreover, equality holds only if

(1) $\left\langle R m^{*}(\boldsymbol{J} v, v) v, \boldsymbol{J} v\right\rangle=0$,

(2) $\sum_{i=1}^{2 n-2}\left\langle R m^{*}\left(w_{i}, v\right) v, w_{i}\right\rangle=0$,

on $B_{x}(R)$.

Theorem 1.2. Assume that the Tanaka-Webster curvature Rm* of the Sasakian manifold satisfies

(1) $\left\langle R m^{*}(\boldsymbol{J} v, v) v, \boldsymbol{J} v\right\rangle \geq 4|v|^{4}$,

(2) $\sum_{i=1}^{2 n-2}\left\langle R m^{*}\left(w_{i}, v\right) v, w_{i}\right\rangle \geq(2 n-2)|v|^{2}$,

where $v$ is any vector in $\mathcal{D}$ and $w_{1}, \ldots, w_{2 n-2}$ in an orthonormal frame of $\left\{v_{0}, v, \boldsymbol{J} v\right\}^{\perp}$. Then

$$
\eta\left(B_{x}(R)\right) \leq \eta_{H}\left(B_{H}(R)\right) .
$$

Moreover, equality holds only if

(1) $\left\langle R m^{*}(\boldsymbol{J} v, v) v, \boldsymbol{J} v\right\rangle=4|v|^{4}$,

(2) $\sum_{i=1}^{2 n-2}\left\langle R m^{*}\left(w_{i}, v\right) v, w_{i}\right\rangle=(2 n-2)|v|^{2}$, on $B_{x}(R)$.

A Laplacian type comparison theorem generalizing the one in [1] also holds. Recall that sub-Laplacian $\Delta_{H}$ is defined by

$$
\Delta f=\sum_{i=1}^{2 n}\left\langle\nabla_{v_{i}} \nabla f, v_{i}\right\rangle
$$

where $v_{1}, \ldots, v_{2 n}$ is an orthonormal frame in $\mathcal{D}$.

Theorem 1.3. Let $x_{0}$ be a point in $M$ and let $d(x):=d\left(x_{0}, x\right)$ be the sub-Riemannian distance from the point $x_{0}$. Assume that the TanakaWebster curvature Rm* of the Sasakian manifold satisfies

(1) $\left\langle R m^{*}(\boldsymbol{J} v, v) v, \boldsymbol{J} v\right\rangle \geq k_{1}|v|^{4}$

(2) $\sum_{i=1}^{2 n-2}\left\langle R m^{*}\left(w_{i}, v\right) v, w_{i}\right\rangle \geq(2 n-2) k_{2}|v|^{2}$,

for some constants $k_{1}$ and $k_{2}$, where $v$ is any vector in $\mathcal{D}$ and $w_{1}, \ldots, w_{2 n-2}$ in an orthonormal frame of $\left\{v_{0}, v, \boldsymbol{J} v\right\}^{\perp}$. Then

$$
\Delta_{H} d \leq h\left(d, v_{0}(d)\right)
$$

where $\mathfrak{k}_{1}(r, z)=z^{2}+k_{1} r^{2}, \mathfrak{k}_{2}(r, z)=\frac{1}{4} z^{2}+k_{2} r^{2}$, and

$$
h(r, z)=\frac{\sqrt{\mathfrak{k}_{1}}\left(\sin \left(\sqrt{\mathfrak{k}_{1}}-\sqrt{\mathfrak{k}_{1}} \cos \left(\sqrt{\mathfrak{k}_{1}}\right)\right)\right.}{r\left(2-2 \cos \left(\sqrt{\mathfrak{k}_{1}}\right)-\sqrt{\mathfrak{k}_{1}} \sin \left(\sqrt{\mathfrak{k}_{1}}\right)\right)}+\frac{(2 n-1) \sqrt{\mathfrak{k}_{2}} \cot \left(\sqrt{\mathfrak{k}_{2}}\right)}{r}
$$




$$
\begin{aligned}
& \text { if } \mathfrak{k}_{1} \geq 0 \text { and } \mathfrak{k}_{2} \geq 0, \\
& \begin{aligned}
h(r, z)=\frac{\left.\sqrt{\mathfrak{k}_{1}}\left(\sqrt{\mathfrak{k}_{1}} \cosh \left(\sqrt{\mathfrak{k}_{1}}\right)\right)-\sinh \left(\sqrt{\mathfrak{k}_{1}}\right)\right)}{r\left(2-2 \cosh \left(\sqrt{-\mathfrak{k}_{1}}\right)+\sqrt{-\mathfrak{k}_{1}} \sinh \left(\sqrt{-\mathfrak{k}_{1}}\right)\right)}+\frac{(2 n-1) \sqrt{\mathfrak{k}_{2}} \cot \left(\sqrt{\mathfrak{k}_{2}}\right)}{r} \\
\text { if } \mathfrak{k}_{1} \geq 0 \text { and } \mathfrak{k}_{2} \leq 0, \\
h(r, z)=\frac{\sqrt{\mathfrak{k}_{1}}\left(\sin \left(\sqrt{\mathfrak{k}_{1}}-\sqrt{\mathfrak{k}_{1}} \cos \left(\sqrt{\mathfrak{k}_{1}}\right)\right)\right.}{r\left(2-2 \cos \left(\sqrt{\mathfrak{k}_{1}}\right)-\sqrt{\mathfrak{k}_{1}} \sin \left(\sqrt{\mathfrak{k}_{1}}\right)\right)}+\frac{(2 n-1) \sqrt{\mathfrak{k}_{2}} \operatorname{coth}\left(\sqrt{\mathfrak{k}_{2}}\right)}{r}
\end{aligned}
\end{aligned}
$$$$
\text { if } \mathfrak{k}_{1} \leq 0 \text { and } \mathfrak{k}_{2} \geq 0 \text {, }
$$$$
h(r, z)=\frac{\left.\sqrt{\mathfrak{k}_{1}}\left(\sqrt{\mathfrak{k}_{1}} \cosh \left(\sqrt{\mathfrak{k}_{1}}\right)\right)-\sinh \left(\sqrt{\mathfrak{k}_{1}}\right)\right)}{r\left(2-2 \cosh \left(\sqrt{-\mathfrak{k}_{1}}\right)+\sqrt{-\mathfrak{k}_{1}} \sinh \left(\sqrt{-\mathfrak{k}_{1}}\right)\right)}+\frac{(2 n-1) \sqrt{\mathfrak{k}_{2}} \operatorname{coth}\left(\sqrt{\mathfrak{k}_{2}}\right)}{r}
$$

if $\mathfrak{k}_{1} \leq 0$ and $\mathfrak{k}_{2} \leq 0$.

A version of Hessian comparison theorem as in [1] also hold. The proof is very similar to and simpler than that of Theorem 1.3. We omit the statement since it is rather lengthy.

The paper is organized as follows. In section 2, we recall the construction of the canonical frame introduced in [8]. In section 3, we recall the definition of Sasakian manifolds. We also recall the definition of parallel adapted frame introduced in [7] which simplifies the computation of the canonical frame, which is done in section 5. In section 6, we prove a first conjugate time estimate under the lower bounds on the Tanaka-Webster curvature. In section 7, we discuss the Heisenberg group, the complex Hopf fibration, and their sub-Riemannian cut locus. The volume estimate and the proof of Theorem 1.1 and 1.2 are done in section 8 . Finally, section 9 is devoted to the proof of Theorem 1.3 .

\section{Canonical frames and Curvatures of a Jacobi Curve}

In this section, we recall how to construct canonical frames and define the curvature of a curve in Lagrangian Grassmannian. We will only do the construction in our simplified setting. For the most general discussion, see [8]. For completeness, we will also include the full proof of the results in our case.

Let $t \mapsto J(t)$ be a curve in the Lagrangian Grassmannian of a symplectic vector space $\mathfrak{V}$. Let $g_{t}^{0}$ be the bilinear form on $J(t)$ defined by

$$
g_{t}^{0}(e, e)=\omega(\dot{e}(t), e)
$$

where $e(\cdot)$ is any curve in $J$ such that $e(t)=e$. 
Assume that the curve $J$ is monotone which means that $g_{t}^{0}$ is nonnegative definite for each $t$. Let $J^{-1}, J^{1}$, and $J^{2}$ be defined by

$$
\begin{aligned}
& J^{-2}(t)=\{e(t) \mid \dot{e}(t), \ddot{e}(t) \in J(t)\}, \\
& J^{-1}(t)=\{e(t) \mid \dot{e}(t) \in J(t)\}, \\
& J^{1}(t)=\operatorname{span}\{e(t), \dot{e}(t) \mid e(\cdot) \in J\}=\left(J^{-1}\right)^{\angle} \\
& J^{2}(t)=\operatorname{span}\{e(t), \dot{e}(t), \ddot{e}(t) \mid e(\cdot) \in J\}=\left(J^{-2}\right)^{\angle}
\end{aligned}
$$

where the superscript $W^{<}$denotes the symplectic complement of the subspace $W$.

We will consider the case $J^{1} \neq \mathfrak{V}$ and $J^{2}=\mathfrak{V}$. Assume that $J$ and $J^{-1}$ have dimensions $N$ and $k$, respectively.

Theorem 2.1. [8] Under the above assumptions, there exists a family of frames $E^{1}(t)=\left(E_{1}^{1}(t), \ldots, E_{k}^{1}(t)\right)^{T}, E^{2}(t)=\left(E_{1}^{2}(t), \ldots, E_{k}^{2}(t)\right)^{T}$, $E^{3}(t)=\left(E_{1}^{3}(t), \ldots, E_{N-2 k}^{3}(t)\right)^{T}, F^{1}(t)=\left(F_{1}^{1}(t), \ldots, F_{k}^{1}(t)\right)^{T}, F^{2}(t)=$ $\left(F_{1}^{2}(t), \ldots, F_{k}^{2}(t)\right)^{T}, F^{3}(t)=\left(F_{1}^{3}(t), \ldots, F_{N-2 k}^{3}(t)\right)^{T}$ such that

(1) $E(t)=\left(E^{1}(t), E^{2}(t), E^{3}(t)\right)^{T}, F(t)=\left(F^{1}(t), F^{2}(t), F^{3}(t)\right)^{T}$ is a symplectic basis for each $t$,

(2) $E^{1}(t)$ is a basis of $J^{-1}(t)$,

(3) $\dot{E}(t)=C_{1} E(t)+C_{2} F(t), \quad \dot{F}(t)=-R(t) E(t)-C_{1}^{T} F(t)$,

where

$$
\begin{aligned}
& C_{1}=\left(\begin{array}{lll}
0 & I & 0 \\
0 & 0 & 0 \\
0 & 0 & 0
\end{array}\right), C_{2}=\left(\begin{array}{ccc}
0 & 0 & 0 \\
0 & I & 0 \\
0 & 0 & I
\end{array}\right), \\
& R(t)=\left(\begin{array}{ccc}
R^{11}(t) & 0 & R^{13}(t) \\
0 & R^{22}(t) & R^{23}(t) \\
R^{31}(t) & R^{32}(t) & R^{33}(t)
\end{array}\right)
\end{aligned}
$$

and $R(t)$ is symmetric.

The frame $\left(E^{1}, E^{2}, E^{3}, F^{1}, F^{2}, F^{3}\right)$ is called a canonical frame of the curve $J$ and the coefficients $R^{i j}$ are the curvatures of the curve $J$. We also write the above equations as

$$
\begin{aligned}
& \dot{E}^{1}(t)=E^{2}(t), \quad \dot{E}^{2}(t)=F^{2}(t), \quad \dot{E}^{3}(t)=F^{3}(t), \\
& \dot{F}^{1}(t)=-R^{11}(t) E^{1}(t)-R^{13}(t) E^{3}(t), \\
& \dot{F}^{2}(t)=-R^{22}(t) E^{2}(t)-R^{23}(t) E^{3}(t)-F^{1}(t), \\
& \dot{F}^{3}(t)=-R^{31}(t) E^{1}(t)-R^{32}(t) E^{2}(t)-R^{33}(t) E^{3}(t) .
\end{aligned}
$$

Proof. Let $g_{t}^{1}$ be the bilinear form on $J^{-1}(t)$ defined by

$$
g_{t}^{1}(e, e)=\omega(\ddot{e}(t), \dot{e}(t)),
$$


where $e(\cdot)$ is any curve in $J^{-1}$ such that $e(t)=e$.

The bilinear form $g_{t}^{1}$ is well-defined. Indeed, let $e_{1}(\cdot)$ and $e_{2}(\cdot)$ be two curves in $J^{-1}(\cdot)$ such that $e_{1}(t)=e_{2}(t)$. Let $e_{3}(\cdot)$ be a curve in $J^{1}$. Since $J^{-1}$ is the skew-orthogonal complement of $J^{1}$, we have

$$
\omega\left(e_{1}(s)-e_{2}(s), e_{3}(s)\right)=0 .
$$

By differentiating the above expression, we have

$$
\omega\left(\dot{e}_{1}(t)-\dot{e}_{2}(t), e_{3}(t)\right)=0 .
$$

Since $e_{3}(t)$ is arbitrary, we see that $\dot{e}_{1}(t)-\dot{e}_{2}(t)$ is contained in $J^{-1}(t)$. On the other hand, since $\dot{e}_{1}(s)$ and $\dot{e}_{2}(s)$ are contained in $J(s)$ and $J(s)$ is Lagrangian, we have

$$
\omega\left(\dot{e}_{1}(s)-\dot{e}_{2}(s), \dot{e}_{1}(s)\right)=0 .
$$

Since $\ddot{e}_{1}(s)$ and $\ddot{e}_{2}(s)$ are contained in $J^{1}(s)$, we have, by differentiating the above expression,

$$
\omega\left(\ddot{e}_{1}(t), \dot{e}_{1}(t)\right)=\omega\left(\ddot{e}_{2}(t), \dot{e}_{1}(t)\right)=\omega\left(\ddot{e}_{2}(t), \dot{e}_{2}(t)\right)
$$

and $g_{t}^{1}$ is well-defined.

Next, we claim that $g_{t}^{1}$ is an inner product and there exists a family of basis $E^{1}(\cdot)=\left(E_{1}^{1}(\cdot), \ldots, E_{k}^{1}(\cdot)\right)^{T}$ along $J^{-1}(\cdot)$ which is orthonormal with respect to $g^{1}$ such that

$$
\omega\left(\ddot{E}^{1}, \ddot{E}^{1}\right)=0 .
$$

Here if $E=\left(E_{1}, \ldots, E_{k}\right)$ and $F=\left(F_{1}, \ldots, F_{k}\right)$ are two vectors, then $\omega(E, F)$ denotes the matrix with $i j$-th entry equal to $\omega\left(E_{i}, F_{j}\right)$.

Moreover, the family $E^{1}(\cdot)$ is unique up to multiplication by an orthogonal matrix (independent of time $t$ ). Indeed let $\bar{E}(\cdot)$ be a family of basis in $J^{-1}(\cdot)$. Since $J^{-2}=\left(J^{2}\right)^{<}=\{0\}, \dot{\bar{E}}(t)$ is not in $J^{-1}(t)$ which is the kernel of $g_{t}^{0}$. Therefore,

$$
g_{t}^{1}(\bar{E}, \bar{E})=g_{t}^{0}(\dot{\bar{E}}, \dot{\bar{E}})
$$

is positive definite.

Let $\bar{E}^{1}=\left(\bar{E}_{1}^{1}, \ldots, \bar{E}_{k}^{1}\right)^{T}$ be a family of curves in $J^{-1}$ such that

$$
\left(\bar{E}_{1}^{1}(t), \ldots, \bar{E}_{k}^{1}(t)\right)^{T}
$$

is an orthonormal basis of $J^{-1}$ with respect to $g_{t}^{1}$. Then any other such family is given by $E(t)=O(t) \bar{E}(t)$. Therefore,

$$
\begin{aligned}
\omega\left(\frac{d^{2}}{d t^{2}}\left(O E^{1}\right), \frac{d^{2}}{d t^{2}}\left(O E^{1}\right)\right) & =\omega\left(2 \dot{O} \dot{E}^{1}+O \ddot{E}^{1}, 2 \dot{O} \dot{E}^{1}+O \ddot{E}^{1}\right) \\
& =-2 \dot{O} O^{T}+2 O \dot{O}^{T}+O \omega\left(\ddot{E}^{1}, \ddot{E}^{1}\right) O^{T} \\
& =-4 \dot{O} O^{T}+O \omega\left(\ddot{E}^{1}, \ddot{E}^{1}\right) O^{T}
\end{aligned}
$$


Here, the first equality holds since $E^{1}(t)$ is contained in $J^{-1}(t)$ and $\dot{E}^{1}(t), \ddot{E}^{1}(t)$ are contained in $J^{1}(t)$. The second equality holds since $\omega\left(\ddot{E}^{1}(t), \dot{E}^{1}(t)\right)=g_{t}^{1}\left(E^{1}(t), E^{1}(t)\right)=I$ and $\dot{E}^{1}(t)$ is in $J(t)$. The last equality holds since $O(t)$ is orthogonal.

It follows that $E^{1}$ satisfies $\omega\left(\ddot{E}^{1}, \ddot{E}^{1}\right)=0$ if and only if $O$ is a solution to the equation

$$
\dot{O}=\frac{1}{4} O \omega\left(\ddot{E}^{1}, \ddot{E}^{1}\right)
$$

This finishes the construction of $E^{1}(t)$.

Let $E^{2}(t)=\dot{E}^{1}(t)$ and let $F^{2}(t)=\dot{E}^{2}(t)$. By construction, we have $\omega\left(F^{2}(t), E^{2}(t)\right)=g_{t}^{1}\left(E^{1}(t), E^{1}(t)\right)=I$. Since $J(t)$ is Lagrangian, $\omega\left(E^{1}(t), E^{2}(t)\right)=0$. Since $F^{2}(t)$ is contained in $J^{1}(t)$ and $E^{1}(t)$ is contained in $J^{-1}(t), \omega\left(F^{2}(t), E^{1}(t)\right)=0$. By construction, we also have $\omega\left(F^{2}(t), F^{2}(t)\right)=\omega\left(\ddot{E}^{1}(t), \ddot{E}^{1}(t)\right)=0$. Next, we complete $E^{1}(t), E^{2}(t)$ to a basis of $J(t)$ by adding $E^{3}(t)$. Moreover, we can assume that $E^{3}(t)$ satisfies the conditions $g_{t}^{0}\left(E^{3}(t), E^{3}(t)\right)=I, \omega\left(E^{3}(t), E^{1}(t)\right)=$ $\omega\left(E^{3}(t), E^{2}(t)\right)=0, \omega\left(E^{3}(t), F^{2}(t)\right)=0$, and $\omega\left(E^{3}(t), \dot{F}^{2}(t)\right)=0$. Indeed, let us complete $E^{1}, E^{2}$ to a basis of $J$ by adding $\bar{E}^{3}$. Let $E^{3}$ be

$$
E^{3}(t)=O_{3}(t)\left(\bar{E}^{3}(t)-\omega\left(\bar{E}^{3}(t), F^{2}(t)\right) E^{2}(t)-\omega\left(\bar{E}^{3}(t), \dot{F}^{2}(t)\right) E^{1}(t)\right) .
$$

Clearly, we have $\omega\left(E^{3}(t), E^{1}(t)\right)=\omega\left(E^{3}(t), E^{2}(t)\right)=\omega\left(E^{3}(t), F^{2}(t)\right)=$ 0 . We also have

$$
\begin{aligned}
\omega & \left(E^{3}(t), \dot{F}^{2}(t)\right) \\
= & O_{3}(t) \omega\left(\bar{E}^{3}(t), \dot{F}^{2}(t)\right)-O_{3}(t) \omega\left(\bar{E}^{3}(t), F^{2}(t)\right) \omega\left(E^{2}(t), \dot{F}^{2}(t)\right) \\
& +O_{3}(t) \omega\left(\bar{E}^{3}(t), \dot{F}^{2}(t)\right) \omega\left(E^{2}(t), F^{2}(t)\right) \\
= & -\omega\left(\bar{E}^{3}(t), F^{2}(t)\right) \omega\left(E^{2}(t), \dot{F}^{2}(t)\right) \\
= & \omega\left(\bar{E}^{3}(t), F^{2}(t)\right) \omega\left(\ddot{E}^{1}(t), \ddot{E}^{1}(t)\right)=0
\end{aligned}
$$

Finally since the kernel of the bilinear form $g_{t}^{0}$ is $J^{-1}$, we also obtain

$$
g_{t}^{0}\left(E^{3}(t), E^{3}(t)\right)=O_{3}(t) g_{t}^{0}\left(\bar{E}^{3}(t), \bar{E}^{3}(t)\right) O_{3}(t)^{T} .
$$

Since $g_{t}^{0}\left(\bar{E}^{3}(t), \bar{E}^{3}(t)\right)$ is positive definite symmetric, we have

$$
g_{t}^{0}\left(E^{3}(t), E^{3}(t)\right)=I
$$

if we set $O_{3}(t)=g_{t}^{0}\left(\bar{E}^{3}(t), \bar{E}^{3}(t)\right)^{-1 / 2}$.

Next, we show that $E^{3}$ can be chosen such that $\ddot{E}^{3}(t)$ is contained in $J(t)$. Moreover any such $E^{3}$ is unique up to multiplication by an 
orthogonal matrix (independent of time $t$ ). Indeed, let $\bar{E}^{3}$ be a family defined above. Since $\omega\left(\dot{\bar{E}}^{3}(t), E^{1}(t)\right)=0$ Then we have

$$
\omega\left(\ddot{\bar{E}}^{3}(t), E^{1}(t)\right)=-\omega\left(\dot{\bar{E}}^{3}(t), E^{2}(t)\right)=\omega\left(\bar{E}^{3}(t), F^{2}(t)\right)=0 .
$$

Similarly, since $\omega\left(\dot{\bar{E}}^{3}(t), E^{2}(t)\right)=0$, we also have

$$
\omega\left(\ddot{\bar{E}}^{3}(t), E^{2}(t)\right)=-\omega\left(\dot{\bar{E}}^{3}(t), F^{2}(t)\right)=\omega\left(\bar{E}^{3}(t), \dot{F}^{2}(t)\right)=0 .
$$

Let $E^{3}(t)=O(t) \bar{E}^{3}(t)$. Then

$$
\begin{aligned}
\omega\left(\ddot{E}^{3}(t), E^{3}(t)\right) & =\omega\left(\ddot{O}(t) \bar{E}^{3}(t)+2 \dot{O}(t) \dot{\bar{E}}^{3}(t)+O(t) \ddot{\bar{E}}^{3}(t), O(t) \bar{E}^{3}(t)\right) \\
& =2 \dot{O}(t) O(t)^{T}+O(t) \omega\left(\ddot{\bar{E}}^{3}(t), \bar{E}^{3}(t)\right) O(t)^{T} \\
& =2 \dot{O}(t) O(t)^{T}-O(t) \omega\left(\dot{\bar{E}}^{3}(t), \dot{\bar{E}}^{3}(t)\right) O(t)^{T} .
\end{aligned}
$$

Therefore, $E^{3}$ satisfies $\omega\left(\ddot{E}^{3}, E^{3}\right)=0$ if and only if $O$ is a solution of the equation $\dot{O}=\frac{1}{2} O \omega\left(\dot{E}^{3}, \dot{E}^{3}\right)$. This finishes the construction of $E^{3}$.

Let $F^{3}(t)=\dot{E}^{3}(t)$. We can complete $E^{1}, E^{2}, E^{3}, F^{2}, F^{3}$ to a symplectic basis by adding $F^{1}$. Moreover, there is a unique such $F^{1}$ satisfying $\omega\left(\dot{F}^{1}(t), F^{2}(t)\right)=0$. Indeed, suppose we have two ways to complete $E^{1}, E^{2}, E^{3}, F^{2}, F^{3}$ to a symplectic basis, say $\bar{F}^{1}$ and $F^{1}$. Then $F^{1}(t)=\bar{F}^{1}(t)+O(t) E^{1}(t)$ for some matrices $O(t)$. But

$$
\begin{aligned}
\omega\left(\dot{F}^{1}(t), F^{2}(t)\right) & =\omega\left(\dot{\bar{F}}^{1}(t)+\dot{O}(t) E^{1}(t)+O(t) E^{2}(t), F^{2}(t)\right) \\
& =\omega\left(\dot{\bar{F}}^{1}(t), F^{2}(t)\right)-O(t) .
\end{aligned}
$$

Therefore, $\omega\left(\dot{F}^{1}(t), F^{2}(t)\right)=0$ if and only if

$$
O=\omega\left(\dot{\bar{F}}^{1}(t), F^{2}(t)\right) \text {. }
$$

\section{SASAKIAN MANIFOLDS AND PARALLEL ADAPTED FRAMES}

In this section, we recall the definition of Sasakian manifolds and introduce the parallel adapted frames. For the part on Sasakian manifolds, we mainly follow 3. Parallel adapted frames were introduced in [7]. It will be used to simplify some tedious calculations in a way very similar to the use of geodesic normal coordinates in Riemannian geometry.

Recall that a manifold $M$ of dimension $2 n+1$ has an almost contact structure $\left(\mathbf{J}, v_{0}, \alpha_{0}\right)$ if $\mathbf{J}: T M \rightarrow T M$ is a $(1,1)$ tensor, $v_{0}$ is a vector field, and $\alpha_{0}$ is a 1-form satisfying

$$
\mathbf{J}^{2}(v)=-v+\alpha_{0}(v) v_{0} \quad \text { and } \quad \alpha_{0}\left(v_{0}\right)=1
$$


for all tangent vector $v$ in $T M$.

An almost contact structure is normal if the following tensor vanishes

$$
(v, w) \mapsto[\mathbf{J}, \mathbf{J}](v, w)+d \alpha_{0}(v, w) v_{0},
$$

where $[\mathbf{J}, \mathbf{J}]$ is defined by

$$
[\mathbf{J}, \mathbf{J}](v, w)=\mathbf{J}^{2}[v, w]+[\mathbf{J} v, \mathbf{J} w]-\mathbf{J}[\mathbf{J} v, w]-\mathbf{J}[v, \mathbf{J} w] .
$$

A Riemannian metric $\langle\cdot, \cdot\rangle$ is compatible with a given almost contact manifold if

$$
\langle\mathbf{J} v, \mathbf{J} w\rangle=\langle v, w\rangle-\alpha_{0}(v) \alpha_{0}(w)
$$

for all tangent vectors $v$ and $w$ in $T M$.

If, in addition, the Riemannian metric satisfies the condition

$$
\langle v, \mathbf{J} w\rangle=d \alpha_{0}(v, w),
$$

then we say that the metric is associated to the given almost contact structure.

Finally, a Sasakian manifold is a normal almost contact manifold with an associated Riemannian metric. The following results can be found in [3]. Since the sign conventions in [3] is different, we include the proof in the appendix.

Theorem 3.1. The followings hold on a Sasakian manifold $\left(\boldsymbol{J}, v_{0}, \alpha_{0}, g=\right.$ $\langle\cdot, \cdot\rangle)$

(1) $\mathcal{L}_{v_{0}}(\boldsymbol{J})=0$

(2) $\nabla_{v_{0}} v_{0}=0$

(3) $\mathcal{L}_{v_{0}} g=0$

(4) $\boldsymbol{J}=-2 \nabla v_{0}$,

where $\nabla$ denotes the Levi-Civita connection.

Theorem 3.2. An almost contact metric manifold $\left(\boldsymbol{J}, v_{0}, \alpha_{0},\langle\cdot, \cdot\rangle\right)$ is Sasakian if and only if it satisfies

$$
\left(\nabla_{v} \boldsymbol{J}\right) w=\frac{1}{2}\langle v, w\rangle v_{0}-\frac{1}{2} \alpha_{0}(w) v
$$

for all tangent vectors $v$ and $w$.

Let $\mathrm{Rm}$ denotes the Riemann curvature tensor.

Theorem 3.3. Assume that the almost contact metric manifold $\left(\boldsymbol{J}, v_{0}, \alpha_{0},\langle\cdot, \cdot\rangle\right)$ is Sasakian. Then

$$
R m(X, Y) v_{0}=\frac{1}{4} \alpha_{0}(Y) X-\frac{1}{4} \alpha_{0}(X) Y
$$


The Tanaka connection $\nabla^{*}$ is defined by

$$
\nabla_{X}^{*} Y=\nabla_{X} Y+\frac{1}{2} \alpha_{0}(X) \mathbf{J} Y-\alpha_{0}(Y) \nabla_{X} v_{0}+\nabla_{X} \alpha_{0}(Y) v_{0}
$$

The corresponding curvature operator is denoted by $\mathrm{Rm}^{*}$ and we call it Tanaka-Webster curvature.

Theorem 3.4. Assume that the tangent vectors $X, Y$, and $Z$ are contained in ker $\alpha_{0}$. Then

$$
R m^{*}(X, Y) Z=(R m(X, Y) Z)^{h}+\left\langle Z, \nabla_{Y} v_{0}\right\rangle \nabla_{X} v_{0}-\left\langle Z, \nabla_{X} v_{0}\right\rangle \nabla_{Y} v_{0},
$$

where the superscript $X^{h}$ denotes the the component of $X$ in $\operatorname{ker} \alpha_{0}$.

If the manifold is Sasakian, then

$$
R m^{*}(X, Y) Z=(R m(X, Y) Z)^{h}+\frac{1}{4}\langle Z, \boldsymbol{J} Y\rangle \boldsymbol{J} X-\frac{1}{4}\langle Z, \boldsymbol{J} X\rangle \boldsymbol{J} Y .
$$

Finally, we introduce the parallel adapted frames.

Lemma 3.5. Let $v_{0}$ be a vector field in a Riemannian manifold $M$. Let $\gamma:[0, T] \rightarrow M$ be a curve in the Riemannian manifold $M$ and let $v_{0}, \ldots, v_{2 n}$ be an orthonormal frame at $x:=\gamma(0)$. Then there is a orthonormal frame $v_{0}(t):=v_{0}(\gamma(t)), v_{1}(t), \ldots, v_{2 n}(t)$ such that

(1) $v_{i}(0)=v_{i}$ and

(2) $\dot{v}_{i}(t)$ is contained in $\mathbb{R} v_{0}$ for each $t$, where $\dot{v}_{i}(t)$ denotes the covariant derivative of $v(\cdot)$ along $\gamma(\cdot)$ and $i=$ $1, \ldots, 2 n$.

The moving frame defined in Lemma 3.5 is called parallel adapted frame introduced in [7]. Using this frame, we obtain the following convenient local frame.

Lemma 3.6. Suppose that $\left(\boldsymbol{J}, v_{0}, \alpha_{0}\right)$ defines an almost contact structure on $M$ and let $\langle\cdot, \cdot\rangle$ be an associated Riemannian metric. For each point $x$ in $M$, there is orthonormal frame $v_{0}, v_{1}, \ldots, v_{2 n}$ defined in a neighborhood of $x$ such that the following conditions hold at $x$.

(1) $\nabla_{v_{i}} v_{j}=-\left\langle\nabla_{v_{i}} v_{0}, v_{j}\right\rangle v_{0}$,

(2) $\nabla_{v_{i}} v_{0}=\sum_{j \neq 0}\left\langle\nabla_{v_{i}} v_{0}, v_{j}\right\rangle v_{j}$

(3) $\nabla_{v_{0}} v_{i}=\nabla_{v_{0}} v_{0}=0$, where $i, j=1, \ldots, 2 n$.

If, in addition, the manifold $M$ together with $\left(\boldsymbol{J}, v_{0}, \alpha_{0}\right)$ is Sasakian, then the followings hold at $x$.

(1) $\nabla_{v_{i}} v_{j}=\frac{1}{2}\left\langle\boldsymbol{J} v_{i}, v_{j}\right\rangle v_{0}$

(2) $\nabla_{v_{i}} v_{0}=-\frac{1}{2} \boldsymbol{J} v_{i}$

(3) $\nabla_{v_{0}} v_{i}=\nabla_{v_{0}} v_{0}=0$ 
The following will be useful for the later sections.

Lemma 3.7. Assume that $\left(M, \boldsymbol{J}, v_{0}, \alpha_{0},\langle\cdot, \cdot\rangle\right)$ is Sasakian. Let $v_{0}, v_{1}, \ldots, v_{2 n}$ be a frame defined by Lemma [3.6, let $\boldsymbol{J}_{i j}=\left\langle\boldsymbol{J} v_{i}, v_{j}\right\rangle$, and let $\Gamma_{i j}^{k}=$ $\left\langle\nabla_{v_{i}} v_{j}, v_{k}\right\rangle$. Then the following holds at $x$

(1) $\Gamma_{00}^{i}=\Gamma_{0 i}^{0}=\Gamma_{i 0}^{0}=0$,

(2) $\Gamma_{i j}^{0}=-\Gamma_{j i}^{0}=\frac{1}{2} \boldsymbol{J}_{i j}$

(3) $v_{k} \boldsymbol{J}_{i j}=0$ if $i, j, k \neq 0$,

(4) $R m\left(v_{i}, v_{j}\right) v_{k}=\sum_{s \neq 0}\left(\left(v_{i} \Gamma_{j k}^{s}\right)-\left(v_{j} \Gamma_{i k}^{s}\right)-\frac{1}{4} \boldsymbol{J}_{j k} \boldsymbol{J}_{i s}+\frac{1}{4} \boldsymbol{J}_{i k} \boldsymbol{J}_{j s}\right) v_{s}$ if $i, j, k \neq 0$.

Proof of Lemma 3.5. Let $w_{0}(t):=v_{0}(\gamma(t)), w_{1}(t), \ldots, w_{n}(t)$ be an orthonormal frame defined along $\gamma(\cdot)$. Let $O(\cdot)$ be a family of $2 n \times 2 n$ orthogonal matrices and let $K_{i j}=\left\langle\dot{w}_{i}(t), w_{j}(t)\right\rangle$, and let $v_{i}(t):=$ $\sum_{j=1}^{2 n} O_{i j}(t) w_{j}(t)$. By differentiating with respect to time $t$, we have

$$
\left\langle\dot{v}_{i}(t), v_{j}(t)\right\rangle=\sum_{k, l}\left(\dot{O}_{i k}(t)+O_{i l}(t) K_{l k}(t)\right) O_{j k}(t) .
$$

Therefore, by setting $\dot{O}(t)+O(t) K(t)=0$, we have that $\dot{v}_{i}$ is vertical.

Proof of Lemma 3.6. We fix a neighborhood of $x$ on which any point in it can be connected to $x$ by a unique geodesic. We then define $v_{i}$ to be the vector field on this neighborhood such that $v_{i}(\gamma(t))$ is a parallel adapted frame along each geodesic $\gamma(\cdot)$ with $\gamma(0)=x$. It follows immediately that $\nabla_{v_{k}} v_{i}$ is vertical, where $i=1, \ldots, 2 n$ and $k=0, \ldots, 2 n$. Therefore,

$$
\nabla_{v_{k}} v_{i}=\left\langle\nabla_{v_{k}} v_{i}, v_{0}\right\rangle v_{0}=-\left\langle v_{i}, \nabla_{v_{k}} v_{0}\right\rangle v_{0}
$$

If $k=0$, then

$$
0=d \alpha_{0}\left(v_{0}, v_{i}\right)=-\alpha_{0}\left(\left[v_{0}, v_{i}\right]\right)=\left\langle v_{0}, \nabla_{v_{0}} v_{i}\right\rangle-\left\langle v_{0}, \nabla_{v_{i}} v_{0}\right\rangle .
$$

Since $\left|v_{0}\right|=1$, we also have

$$
\left\langle v_{0}, \nabla_{v_{0}} v_{i}\right\rangle=\left\langle\nabla_{v_{i}} v_{0}, v_{0}\right\rangle=0
$$

and hence $\nabla_{v_{0}} v_{i}=0$.

It also follows that $\left\langle\nabla_{v_{0}} v_{0}, v_{i}\right\rangle=-\left\langle v_{0}, \nabla_{v_{0}} v_{i}\right\rangle=0$. Therefore, $\nabla_{v_{0}} v_{0}=0$. The second part follows from $\left\langle\nabla_{v_{i}} v_{0}, v_{j}\right\rangle=-\left\langle\mathbf{J} v_{i}, v_{j}\right\rangle$ for Sasakian manifolds.

Proof of Lemma 3.7. It is clear that $\Gamma_{i 0}^{0}=0$. Since $\nabla_{v_{0}} v_{0}=0$,

$$
0=\left\langle\nabla_{v_{0}} v_{0}, v_{i}\right\rangle=\Gamma_{00}^{i}=-\Gamma_{0 i}^{0}=0 .
$$

Since $\mathcal{L}_{v_{0}} g=0$,

$$
0=\mathcal{L}_{v_{0}} g\left(v_{i}, v_{j}\right)=-\left\langle v_{i},\left[v_{0}, v_{j}\right]\right\rangle-\left\langle\left[v_{0}, v_{i}\right], v_{j}\right\rangle=-\Gamma_{j i}^{0}-\Gamma_{i j}^{0} .
$$


Since the Riemannian metric is associated to the almost contact structure,

$$
\mathbf{J}_{j i}=\left\langle v_{i}, \mathbf{J} v_{j}\right\rangle=d \alpha_{0}\left(v_{i}, v_{j}\right)=-\alpha_{0}\left(\left[v_{i}, v_{j}\right]\right)=-\left(\Gamma_{i j}^{0}-\Gamma_{j i}^{0}\right)=2 \Gamma_{j i}^{0} .
$$

The third relation follows from the property of the frame $v_{0}, \ldots, v_{2 n}$ and Theorem 3.2 .

Finally, we have

$$
\begin{aligned}
& \operatorname{Rm}\left(v_{i}, v_{j}\right) v_{k}=\nabla_{v_{i}} \nabla_{v_{j}} v_{k}-\nabla_{v_{j}} \nabla_{v_{i}} v_{k}-\nabla_{\left[v_{i}, v_{j}\right]} v_{k} \\
& =\sum_{l}\left(v_{i} \Gamma_{j k}^{l}\right) v_{l}+\sum_{l, s} \Gamma_{j k}^{l} \Gamma_{i l}^{s} v_{s}-\sum_{l}\left(v_{j} \Gamma_{i k}^{l}\right) v_{l} \\
& -\sum_{l, s} \Gamma_{i k}^{l} \Gamma_{j l}^{s} v_{s}-\sum_{l, s} \Gamma_{i j}^{l} \Gamma_{l k}^{s} v_{s}+\sum_{l, s} \Gamma_{j i}^{l} \Gamma_{l k}^{s} v_{s} \\
& =\sum_{s \neq 0}\left(\left(v_{i} \Gamma_{j k}^{s}\right)-\left(v_{j} \Gamma_{i k}^{s}\right)-\frac{1}{4} \mathbf{J}_{j k} \mathbf{J}_{i s}+\frac{1}{4} \mathbf{J}_{i k} \mathbf{J}_{j s}\right) v_{s}
\end{aligned}
$$

\section{Sub-Riemannian GeOdesic Flows And JaCOBi CURVES}

In this section, we give a quick review on some basic notions in subRiemannian geometry. In particular, we will introduce Jacobi curves corresponding to the sub-Riemannian geodesic flow and its induced geometric structures.

A sub-Riemannian manifold is a triple $(M, \mathcal{D},\langle\cdot, \cdot\rangle)$, where $M$ is a manifold of dimension $n, \mathcal{D}$ is a distribution (sub-bundle of the tangent bundle $T M$ ), and $\langle\cdot, \cdot\rangle$ is a sub-Riemannian metric (smoothly varying inner product defined on $\mathcal{D}$ ). Assuming that the manifold $M$ is connected and the distribution $\mathcal{D}$ satisfies the Hörmander condition (the sections of $\mathcal{D}$ and their iterated Lie brackets span each tangent space, also called "bracket-generating" condition). Then, by Chow-Rashevskii Theorem, any two given points on the manifold $M$ can be connected by a horizontal curve (a curve which is almost everywhere tangent to $\mathcal{D})$. Therefore, we can define the sub-Riemannian distance $d$ as

$$
d\left(x_{0}, x_{1}\right)=\inf _{\gamma \in \Gamma} l(\gamma),
$$

where the infimum is taken over the set $\Gamma$ of all horizontal paths $\gamma:[0,1] \rightarrow M$ satisfying $\gamma(0)=x_{0}$ and $\gamma(1)=x_{1}$. The minimizers of (4.1) are called length minimizing geodesics (or simply geodesics). As in the Riemannian case, reparametrizations of a geodesic are also geodesics. Therefore, we assume that all geodesics have constant speed. 
These constant speed geodesics are also minimizers of the kinetic energy functional

$$
\inf _{\gamma \in \Gamma} \int_{0}^{1} \frac{1}{2}|\dot{\gamma}(t)|^{2} d t,
$$

where $|\cdot|$ denotes the norm w.r.t. the sub-Riemannian metric.

Let $H: T^{*} M \rightarrow \mathbb{R}$ be the Hamiltonian defined by the Legendre transform:

$$
H(x, p)=\sup _{v \in \mathcal{D}}\left(p(v)-\frac{1}{2}|v|^{2}\right)
$$

and let

$$
\vec{H}=\sum_{i=1}^{n}\left(H_{p_{i}} \partial_{x_{i}}-H_{x_{i}} \partial_{p_{i}}\right)
$$

be the Hamiltonian vector field. Assume, through out this paper, that the vector field $\vec{H}$ defines a complete flow which is denoted by $e^{t \vec{H}}$. The projections of the trajectories of $e^{t \vec{H}}$ to the manifold $M$ give minimizers of (4.2).

In this paper, we assume that the sub-Riemannian structure is given by a Sasakian manifold. More precisely, assume that the almost contact structure $\left(\mathbf{J}, v_{0}, \alpha_{0}\right)$ together with the Riemannian structure $\langle\cdot, \cdot\rangle$ form a Sasakian manifold. The distribution $\mathcal{D}$ is given by $\mathcal{D}=\operatorname{ker} \alpha_{0}$ and the sub-Riemannian metric is given by the restriction of the Riemannian metric to $\mathcal{D}$. In this case all minimizers of (4.2) are given by the projections of the trajectories of $e^{t \vec{H}}$ (see [10] for more detail).

Next, we discuss a sub-Riemannian analogue of Jacobi fields. Let $\omega$ be the symplectic form on the cotangent bundle $T^{*} M$ defined in local coordinates $\left(x_{1}, \ldots, x_{2 n+1}, p_{1}, \ldots, p_{2 n+1}\right)$ by

$$
\omega=\sum_{i=1}^{2 n+1} d p_{i} \wedge d x_{i} .
$$

Let $\pi: T^{*} M \rightarrow M$ be the canonical projection and let $\mathcal{V}$ be the vertical sub-bundle of the cotangent bundle $T^{*} M$ defined by

$$
\mathcal{V}_{(x, p)}=\left\{v \in T_{(x, p)} T^{*} M \mid \pi_{*}(v)=0\right\} .
$$

The family of Lagrangian subspaces

$$
\mathfrak{J}_{(x, p)}(t):=e_{*}^{-t \vec{H}}\left(\mathcal{V}_{e^{t \vec{H}}(x, p)}\right)
$$

defined a curve in the Lagrangian Grassmannian of $T_{(x, p)} T^{*} M$, called the Jacobi curve at $(x, p)$ of the flow $e^{t \vec{H}}$.

Assuming that the manifold is Sasakian. Then Theorem 2.1 applies and we let $E^{1}(t), E^{2}(t), E^{3}(t), F^{1}(t), F^{2}(t), F^{3}(t)$ be a canonical frame 
of $\mathfrak{J}_{(x, p)}$. This defines a splitting of the vertical space $\mathcal{V}_{(x, p)}$ and the cotangent space $T_{(x, p)} T^{*} M$. More precisely, let

$$
\begin{array}{lll}
\mathcal{V}_{1}=\operatorname{span}\left\{E^{1}(0)\right\}, & \mathcal{V}_{2}=\operatorname{span}\left\{E^{2}(0)\right\}, & \mathcal{V}_{3}=\operatorname{span}\left\{E^{3}(0)\right\} \\
\mathcal{H}_{1}=\operatorname{span}\left\{F^{1}(0)\right\}, & \mathcal{H}_{2}=\operatorname{span}\left\{F^{2}(0)\right\}, & \mathcal{H}_{3}=\operatorname{span}\left\{F^{3}(0)\right\} .
\end{array}
$$

Then $\mathcal{V}_{(x, p)}=\mathcal{V}_{1} \oplus \mathcal{V}_{2} \oplus \mathcal{V}_{3}$ and $T_{(x, p)} T^{*} M=\mathcal{V}_{1} \oplus \mathcal{V}_{2} \oplus \mathcal{V}_{3} \oplus \mathcal{H}_{1} \oplus \mathcal{H}_{2} \oplus \mathcal{H}_{3}$ Note that $\mathcal{V}_{1}, \mathcal{V}_{2}, \mathcal{H}_{1}$, and $\mathcal{H}_{2}$ are all 1-dimensional. $\mathcal{V}_{3}$ and $\mathcal{H}_{3}$ are $(2 n-2)$-dimensional. Let $\alpha$ and $h$ be, respectively, a 1 -form and a function on $T^{*} M$. Let $\vec{\alpha}$ and $\vec{h}$ be the vector fields defined, respectively, by

$$
\omega(\vec{\alpha}, \cdot)=-\alpha \quad \text { and } \quad \omega(\vec{h}, \cdot)=-d h .
$$

Theorem 4.1. Let $x$ be in $M$. The above splitting of the cotangent bundle is given by the followings

(1) $\mathcal{V}_{1}=\operatorname{span}\left\{\vec{\alpha}_{0}\right\}$

(2) $\mathcal{V}_{2}=\operatorname{span}\left\{\sum_{k, l \neq 0} h_{k} \boldsymbol{J}_{k l} \vec{\alpha}_{l}\right\}$

(3) $\mathcal{V}_{3}=\operatorname{span}\left\{\sum_{b} a_{b} \vec{\alpha}_{b} \mid \sum_{j, k \neq 0} a_{k} h_{j} \boldsymbol{J}_{k j}=0\right.$ and $\left.a_{0}=\frac{h_{0}}{2 H} \sum_{k \neq 0} a_{k} h_{k}\right\}$,

(4) $\mathcal{H}_{1}=\operatorname{span}\left\{2 H \vec{h}_{0}-h_{0} \vec{H}\right\}$,

(5) $\mathcal{H}_{2}=\operatorname{span}\left\{h_{0} \sum_{k \neq 0} h_{k} \vec{\alpha}_{k}-\sum_{j, k \neq 0} h_{j} \boldsymbol{J}_{j k} \vec{h}_{k}-H \vec{\alpha}_{0}\right.$

$$
\left.-\sum_{j, k, l \neq 0} h_{j} h_{l} \Gamma_{0 l}^{k} \boldsymbol{J}_{j k} \vec{\alpha}_{0}-\sum_{j, k, l, s \neq 0} h_{j} h_{l} \boldsymbol{J}_{j s} \Gamma_{k l}^{s} \vec{\alpha}_{k}\right\},
$$

(6) $\mathcal{H}_{3}=\left\{\sum_{i \neq 0} a_{i} \vec{h}_{i}+\sum_{a} c_{a} \vec{\alpha}_{a} \mid \sum_{j, k \neq 0} a_{k} h_{j} \boldsymbol{J}_{k j}=0\right.$,

$$
\begin{aligned}
& a_{0}=\frac{h_{0}}{2 H} \sum_{k \neq 0} a_{k} h_{k}, c_{0}=\sum_{i, j \neq 0} a_{i} h_{j} \Gamma_{0 j}^{i}, \\
& \left.c_{k}=\sum_{j \neq 0}\left(\frac{1}{2} a_{j} \boldsymbol{J}_{j k} h_{0}-\frac{1}{2} a_{0} h_{j} \boldsymbol{J}_{j k}+\sum_{i \neq 0} a_{i} h_{j} \Gamma_{k j}^{i}\right)\right\},
\end{aligned}
$$

where $v_{0}, v_{1}, \ldots, v_{2 n}$ is a local frame defined in a neighborhood of a point $x$ by Lemma 3.6, $\boldsymbol{J}_{i j}=\left\langle\boldsymbol{J} v_{i}, v_{j}\right\rangle$.

The vertical splitting can be written in a coordinate free way. For this, we identify the tangent bundle $T M$ with the vertical bundle $\mathcal{V}$ using the Riemannian metric via

$$
v \in T M \rightarrow \alpha(\cdot)=\langle v, \cdot\rangle \in T^{*} M \rightarrow-\vec{\alpha} \in \text { ver }
$$

Under this identification, we have

Theorem 4.2. Let $x$ be in $M$. The above splitting of the cotangent bundle is given by the followings

(1) $\mathcal{V}_{1}=\mathbb{R} v_{0}$

(2) $\mathcal{V}_{2}=\mathbb{R} \boldsymbol{J}_{p^{h}}$

(3) $\mathcal{V}_{3}=\mathbb{R}\left(p^{h}+p\left(v_{0}\right) v_{0}\right) \oplus\left\{v \mid\left\langle v, p^{h}\right\rangle=\left\langle v, \boldsymbol{J} p^{h}\right\rangle=\left\langle v, v_{0}\right\rangle=0\right\}$.

(4) $\pi_{*} \mathcal{H}_{1}=\mathbb{R}\left(\left|p^{h}\right|^{2} v_{0}-p\left(v_{0}\right) p^{h}\right)$,

(5) $\pi_{*} \mathcal{H}_{2}=\mathbb{R} \boldsymbol{J}^{h}$,

(6) $\pi_{*} \mathcal{H}_{3}=\left\{X \mid\left\langle X, \boldsymbol{J}_{p^{h}}\right\rangle=\left\langle X, v_{0}\right\rangle=0\right\}$, 
where $p^{h}$ is the vector in $\operatorname{ker} \alpha_{0}$ defined by $p(v)=\left\langle p^{h}, v\right\rangle$ and $v$ ranges over vectors in $\operatorname{ker} \alpha_{0}$.

Under the above identification, we can also define a volume form $\mathfrak{m}$ on $\mathcal{V}$ by $\mathfrak{m}\left(v_{0}, \ldots, v_{2 n}\right)=1$. The Riemannian volume on $M$ is denoted by $\eta$. The proof of Theorem 4.1 also gives

Theorem 4.3. The volume forms $\mathfrak{m}$ and $\eta$ satisfy

(1) $\mathfrak{m}(E(0))=\frac{1}{\left|p^{h}\right|}$,

(2) $\eta\left(\pi_{*} F(0)\right)=\left|p^{h}\right|$.

Proof of Theorem 4.1. Let $v_{0}, v_{1}, \ldots, v_{2 n}$ be the local frame defined in a neighborhood of $x$ by Lemma 3.6. Let $\Gamma_{a b}^{c}$ and $\mathbf{J}_{i j}$ be defined by

$$
\nabla_{v_{a}} v_{b}=\Gamma_{a b}^{c} v_{c} \quad \text { and } \quad \mathbf{J}_{i j}=\left\langle\mathbf{J} v_{i}, v_{j}\right\rangle,
$$

respectively. From now on, we sum over repeated indices. The indices $i, j, k, s, l$ ranges over $1, \ldots, 2 n$ and $a, b, c, d$ ranges over $0, \ldots, 2 n$.

It is clear that $\Gamma_{a b}^{c}=-\Gamma_{a c}^{b}$ wherever it is defined. We also have $\Gamma_{00}^{i}=\Gamma_{0 i}^{0}=\Gamma_{i 0}^{0}=0$. Indeed, since $d \alpha_{0}\left(v_{0}, v_{i}\right)=0$, we have

$$
0=\alpha_{0}\left(\left[v_{0}, v_{i}\right]\right)=\Gamma_{0 i}^{0}-\Gamma_{i 0}^{0}=\Gamma_{0 i}^{0}=-\Gamma_{00}^{i} .
$$

Since $\left\langle\mathbf{J} v_{i}, v_{j}\right\rangle=-2\left\langle\nabla_{v_{i}} v_{0}, v_{j}\right\rangle$, we have $\mathbf{J}_{i j}=-2 \Gamma_{i 0}^{j}=2 \Gamma_{i j}^{0}$. Let $\alpha_{0}, \ldots, \alpha_{2 n}$ be the dual frame of $v_{0}, \ldots, v_{2 n}$ and let $h_{i}(x, p)=p\left(v_{i}\right)$. Then $\pi^{*} \alpha_{0}, \ldots, \pi^{*} \alpha_{n}, d h_{0}, \ldots, d h_{n}$ forms a local co-frame of the cotangent bundle. We will also denote $\pi^{*} \alpha_{i}$ simply by $\alpha_{i}$.

The proof of the following two lemmas will be postponed to the appendix.

Lemma 4.4. The following relations hold.

(1) $\alpha_{a}\left(\vec{h}_{b}\right)=\delta_{a b}$,

(2) $\left[\vec{\alpha}_{a}, \vec{\alpha}_{b}\right]=0$

(3) $d h_{b}\left(\vec{h}_{c}\right)=\sum_{a}\left(\Gamma_{c b}^{a}-\Gamma_{b c}^{a}\right) h_{a}$,

(4) $\left[\vec{\alpha}_{a}, \vec{h}_{b}\right]=\sum_{c}\left(\Gamma_{b c}^{a}-\Gamma_{c b}^{a}\right) \vec{\alpha}_{c}$,

(5) $\left[\vec{H}, \vec{\alpha}_{i}\right]=\vec{h}_{i}+\sum_{j \neq 0, a} h_{j}\left(\Gamma_{a j}^{i}-\Gamma_{j a}^{i}\right) \vec{\alpha}_{a}$ if $i \neq 0$,

(6) $\left[\vec{H}, \vec{\alpha}_{0}\right]=\sum_{j, k \neq 0} h_{j}\left(\Gamma_{k j}^{0}-\Gamma_{j k}^{0}\right) \vec{\alpha}_{k}=-\sum_{j, k \neq 0} h_{j} \boldsymbol{J}_{j k} \vec{\alpha}_{k}$,

(7) $\left[\vec{H}, \vec{h}_{i}\right]=\sum_{k \neq 0} h_{k}\left[\vec{h}_{k}, \vec{h}_{i}\right]-\sum_{k \neq 0, a} h_{a}\left(\Gamma_{i k}^{a}-\Gamma_{k i}^{a}\right) \vec{h}_{k}$,

(8) $\left[\vec{H},\left[\vec{H}, \vec{\alpha}_{0}\right]\right]=h_{0} \sum_{k \neq 0} h_{k} \vec{\alpha}_{k}-\sum_{k, j \neq 0} h_{j} \boldsymbol{J}_{j k} \vec{h}_{k}$

$-H \vec{\alpha}_{0}-\sum_{j, l, k \neq 0} h_{j} h_{l} \Gamma_{0 l}^{k} \boldsymbol{J}_{j k} \vec{\alpha}_{0}-\sum_{j, l, s, k \neq 0} h_{j} h_{l} \boldsymbol{J}_{j s} \Gamma_{k l}^{s} \vec{\alpha}_{k}$,

(9) $\left[\vec{H},\left[\vec{H}, \vec{\alpha}_{i}\right]\right]=2 \sum_{l, k \neq 0} h_{l} \Gamma_{l i}^{k} \vec{h}_{k}+\sum_{l \neq 0} h_{l} \boldsymbol{J}_{l i} \vec{h}_{0}-\sum_{k \neq 0} h_{0} \boldsymbol{J}_{i k} \vec{h}_{k}$ (mod vertical) when $i \neq 0$,

(10) $\left[\vec{H},\left[\vec{H},\left[\vec{H}, \vec{\alpha}_{0}\right]\right]\right]=h_{0} \vec{H}-2 H \vec{h}_{0}$ (mod vertical). 
Here, the phrase "mod vertical" means the that the difference of the two vectors is contained in the vertical bundle $\mathcal{V}$.

The relations reduce to the following ones at $x$

Lemma 4.5. The following relations hold at $x$.

(1) $d h_{j}\left(\vec{h}_{i}\right)=\boldsymbol{J}_{i j} h_{0}$ if $i \neq 0 \neq j$,

(2) $d h_{j}\left(\vec{h}_{0}\right)=\frac{1}{2} \sum_{k \neq 0} \boldsymbol{J}_{j k} h_{k}$ if $j \neq 0$,

(3) $\left[\vec{\alpha}_{i}, \vec{h}_{j}\right]=\frac{1}{2} \boldsymbol{J}_{i j} \vec{\alpha}_{0}$ if $i \neq 0 \neq j$,

(4) $\left[\vec{\alpha}_{i}, \vec{h}_{0}\right]=\frac{1}{2} \sum_{k \neq 0} \boldsymbol{J}_{k i} \vec{\alpha}_{k}$ if $i \neq 0$,

(5) $\left[\vec{\alpha}_{0}, \vec{h}_{j}\right]=\sum_{k \neq 0} \boldsymbol{J}_{j k} \vec{\alpha}_{k}$ if $j \neq 0$,

(6) $\left[\vec{H}, \vec{\alpha}_{i}\right]=\vec{h}_{i}+\sum_{j \neq 0} h_{j} \boldsymbol{J}_{j i} \vec{\alpha}_{0}$ when $i \neq 0$,

(7) $\left[\vec{H}, \vec{\alpha}_{0}\right]=-\sum_{j, k \neq 0} h_{j} \boldsymbol{J}_{j k} \vec{\alpha}_{k}$,

(8) $\left[\vec{H},\left[\vec{H}, \vec{\alpha}_{0}\right]\right]=h_{0} \sum_{k \neq 0} h_{k} \vec{\alpha}_{k}-\sum_{j, k \neq 0} h_{j} \boldsymbol{J}_{j k} \vec{h}_{k}-H \vec{\alpha}_{0}$,

Now, we apply the above lemmas to prove the theorem. Since $\left[\vec{H}, \vec{\alpha}_{0}\right]$ is vertical, $\vec{\alpha}_{0}$ is in $J^{-1}(0)$. Therefore, $\vec{\alpha}_{0}=f E^{1}(0)$ for some function $f$ on the cotangent bundle. It follows from Theorem 2.1 that

(1) $f E^{2}(0)=\left[\vec{H}, \vec{\alpha}_{0}\right]-(\vec{H} f) E^{1}(0)$,

(2) $f F^{2}(0)=\left[\vec{H},\left[\vec{H}, \vec{\alpha}_{0}\right]\right]-\left(\vec{H}^{2} f\right) E^{1}(0)-2(\vec{H} f) E^{2}(0)$,

(3) $f \dot{F}^{2}(0)=\left[\vec{H},\left[\vec{H},\left[\vec{H}, \vec{\alpha}_{0}\right]\right]\right]-\left(\vec{H}^{3} f\right) E_{1}-3\left(\vec{H}^{2} f\right) E_{2}-3(\vec{H} f) F_{2}$.

By Lemma 4.5, we have

$$
f^{2}=\omega\left(f F^{2}(0), f E^{2}(0)\right)=\sum_{i, l, j, k \neq 0} h_{i} h_{j} \mathbf{J}_{i l} \mathbf{J}_{j k} \omega\left(\vec{h}_{l}, \vec{\alpha}_{k}\right)=2 H .
$$

It follows from this and Lemma 4.4 that

(1) $f E^{2}(0)=-\sum_{k, l \neq 0} h_{k} \mathbf{J}_{k l} \vec{\alpha}_{l}$,

(2) $f F^{2}(0)=h_{0} \sum_{j, k, l \neq 0} h_{k} \vec{\alpha}_{k}-\sum_{j, k \neq 0} h_{j} \mathbf{J}_{j k} \vec{h}_{k}-H \vec{\alpha}_{0}$

$$
-\sum_{j, k, l \neq 0} h_{j} h_{l} \Gamma_{0 l}^{k} \mathbf{J}_{j k} \vec{\alpha}_{0}-\sum_{j, k, l, s \neq 0} h_{j} h_{l} \mathbf{J}_{j s} \Gamma_{k l}^{s} \vec{\alpha}_{k}
$$

(3) $-f F^{1}(0)=f \dot{F}^{2}(0)=h_{0} \vec{H}-2 H \vec{h}_{0}$ (mod vertical).

This gives the characterizations of $\mathcal{V}_{1}, \mathcal{V}_{2}$, and $\mathcal{H}_{2}$.

Suppose that $a_{b} \vec{\alpha}_{b}$ is contained in $\mathcal{V}_{3}$. Since $\mathcal{V}_{3}$ and $\mathcal{H}_{2}$ are skeworthogonal,

$$
-\sum_{j, k \neq 0} a_{k} h_{j} \mathbf{J}_{k j}=\omega\left(a_{b} \vec{\alpha}_{b}, h_{j} \mathbf{J}_{i j} \vec{h}_{i}\right)=0 .
$$

Since $\mathcal{V}_{3}$ and $\mathcal{H}_{1}$ are skew-orthogonal, we also have

$$
0=-\omega\left(a_{b} \vec{\alpha}_{b}, h_{0} \vec{H}-2 H \vec{h}_{0}\right)=h_{0} h_{k} a_{k}-2 H a_{0}
$$

This gives the characterizations of $\mathcal{V}_{3}$. 
It also follows that

$$
\begin{aligned}
& {\left[\vec{H}, a_{0} \vec{\alpha}_{0}+a_{i} \vec{\alpha}_{i}\right]} \\
& =\left(\vec{H} a_{0}\right) \vec{\alpha}_{0}+a_{0}\left[\vec{H}, \vec{\alpha}_{0}\right]+\left(\vec{H} a_{i}\right) \vec{\alpha}_{i}+a_{i}\left[\vec{H}, \vec{\alpha}_{i}\right] \\
& =\left(\vec{H} a_{0}\right) \vec{\alpha}_{0}-a_{0} h_{j} \mathbf{J}_{j k} \vec{\alpha}_{k}+\left(\vec{H} a_{i}\right) \vec{\alpha}_{i}+a_{i} \vec{h}_{i}+a_{i} h_{j}\left(\Gamma_{a j}^{i}-\Gamma_{j a}^{i}\right) \vec{\alpha}_{a} .
\end{aligned}
$$

It follows from the structural equation that $\left[\vec{H}, a_{0} \vec{\alpha}_{0}+a_{i} \vec{\alpha}_{i}\right]$ is contained in $\mathcal{V}_{3} \oplus \mathcal{H}_{3}$. Moreover, if $X_{1}$ and $X_{2}$ are the $\mathcal{V}_{3}$ and $\mathcal{H}_{3}$ parts of $\left[\vec{H}, a_{0} \vec{\alpha}_{0}+a_{i} \vec{\alpha}_{i}\right]$, respectively, then

$$
\pi_{*}\left[\vec{H}, X_{1}\right]=\pi_{*}\left[\vec{H}, X_{2}\right] .
$$

Suppose that $a_{i} \vec{h}_{i}+c_{a} \vec{\alpha}_{a}$ is contained in $\mathcal{H}_{3}$. Then it follows from Lemma 4.4 and the characterization of $\mathcal{V}_{3}$ that

$$
\begin{aligned}
& \pi_{*}\left[\vec{H}, a_{i} \vec{h}_{i}+c_{a} \vec{\alpha}_{a}\right] \\
& =\left(\vec{H} a_{i}\right) v_{i}+a_{i} h_{j}\left[v_{j}, v_{i}\right]-a_{i} \mathbf{J}_{i k} h_{0} v_{k}-a_{i} h_{j}\left(\Gamma_{i k}^{j}-\Gamma_{k i}^{j}\right) v_{k}+c_{i} v_{i} \\
& =\left(\vec{H} a_{i}\right) v_{i}+a_{i} h_{j}\left(\Gamma_{j i}^{k}-\Gamma_{i j}^{k}\right) v_{k}-a_{i} \mathbf{J}_{i k} h_{0} v_{k}-a_{i} h_{j}\left(\Gamma_{i k}^{j}-\Gamma_{k i}^{j}\right) v_{k}+c_{i} v_{i} \\
& =\left(\vec{H} a_{i}\right) v_{i}+a_{i} h_{j}\left(\Gamma_{j i}^{k}+\Gamma_{k i}^{j}\right) v_{k}-a_{i} \mathbf{J}_{i k} h_{0} v_{k}+c_{i} v_{i}
\end{aligned}
$$

and

$$
\begin{aligned}
& \pi_{*}\left[\vec{H},\left(\vec{H} a_{0}\right) \vec{\alpha}_{0}-a_{0} h_{j} \mathbf{J}_{j k} \vec{\alpha}_{k}+\left(\vec{H} a_{i}\right) \vec{\alpha}_{i}+a_{i} h_{j}\left(\Gamma_{a j}^{i}-\Gamma_{j a}^{i}\right) \vec{\alpha}_{a}-c_{a} \vec{\alpha}_{a}\right] \\
& =-a_{0} h_{j} \mathbf{J}_{j k} v_{k}+\left(\vec{H} a_{i}\right) v_{i}+a_{i} h_{j}\left(\Gamma_{k j}^{i}-\Gamma_{j k}^{i}\right) v_{k}-c_{i} v_{i}
\end{aligned}
$$

It follows that

$$
c_{k}=a_{i} h_{j} \Gamma_{k j}^{i}+\frac{1}{2}\left(a_{j} \mathbf{J}_{j k} h_{0}-a_{0} h_{j} \mathbf{J}_{j k}\right) .
$$

It also follows from this that

$$
\begin{aligned}
& \left(\vec{H} a_{0}-c_{0}\right) \vec{\alpha}_{0}-a_{0} h_{j} \mathbf{J}_{j k} \vec{\alpha}_{k}+\left(\vec{H} a_{i}\right) \vec{\alpha}_{i}+a_{i} h_{j}\left(\Gamma_{0 j}^{i}-\Gamma_{j 0}^{i}\right) \vec{\alpha}_{0} \\
& +a_{i} h_{j}\left(\Gamma_{k j}^{i}-\Gamma_{j k}^{i}\right) \vec{\alpha}_{k}-\left(\frac{1}{2} a_{j} \mathbf{J}_{j k} h_{0}-\frac{1}{2} a_{0} h_{j} \mathbf{J}_{j k}+a_{i} h_{j} \Gamma_{k j}^{i}\right) \vec{\alpha}_{k} \\
& =\left(\vec{H} a_{0}-c_{0}+a_{i} h_{j} \Gamma_{0 j}^{i}\right) \vec{\alpha}_{0}+\left(\vec{H} a_{i}\right) \vec{\alpha}_{i}-a_{i} h_{j} \Gamma_{j k}^{i} \vec{\alpha}_{k}-\frac{1}{2}\left(a_{j} \mathbf{J}_{j k} h_{0}+a_{0} h_{j} \mathbf{J}_{j k}\right) \vec{\alpha}_{k}
\end{aligned}
$$

is contained in $\mathcal{V}_{3}$. Therefore,

$$
\begin{aligned}
& 2 H\left(\vec{H} a_{0}-c_{0}+a_{i} h_{j} \Gamma_{0 j}^{i}\right) \\
& =h_{0}\left(\vec{H} a_{k}-\frac{1}{2} a_{j} h_{0} \mathbf{J}_{j k}-\frac{1}{2} a_{0} h_{j} \mathbf{J}_{j k}-a_{i} h_{j} \Gamma_{j k}^{i}\right) h_{k} \\
& =h_{0}\left(\vec{H} a_{k}\right) h_{k}-h_{0} a_{i} \Gamma_{j k}^{i} h_{j} h_{k}
\end{aligned}
$$


On the other hand, it follows from (4.5) that

$$
h_{0} h_{l} h_{s} \Gamma_{l k}^{s} a_{k}+h_{0} h_{k} \vec{H} a_{k}-2 H \vec{H} a_{0}=0 .
$$

Therefore, $c_{0}=a_{i} h_{j} \Gamma_{0 j}^{i}$ and this finishes the characterization of $\mathcal{H}_{3}$.

By the tenth relation in Lemma 4.5 and the structural equation, we can choose a vector in $\mathcal{H}_{1}$ of the form

$$
2 H \vec{h}_{0}-h_{0} \vec{H}+r_{a} \vec{\alpha}_{a}
$$

Since $\mathcal{H}_{1}$ is in the skew orthogonal complement of $\mathcal{H}_{3}$, we have

$$
\begin{aligned}
0 & =\omega\left(a_{i} \vec{h}_{i}+c_{a} \vec{\alpha}_{a}, 2 H \vec{h}_{0}-h_{0} \vec{H}+r_{a} \vec{\alpha}_{a}\right) \\
& =2 H a_{i} d h_{0}\left(\vec{h}_{i}\right)-2 H c_{0}-h_{0} a_{i} d H\left(\vec{h}_{i}\right)+h_{0} c_{j} h_{j}+r_{i} a_{i} \\
& =-2 H a_{i} \Gamma_{0 i}^{s} h_{s}-2 H c_{0}+h_{0} a_{i} h_{j} h_{k} \Gamma_{j i}^{k}+h_{0} c_{j} h_{j}+r_{i} a_{i} \\
& =-2 H a_{i} \Gamma_{0 i}^{s} h_{s}-2 H c_{0}-h_{0} a_{i} h_{j} h_{k} \Gamma_{j k}^{i}+h_{0} a_{i} h_{j} h_{k} \Gamma_{k j}^{i}+r_{i} a_{i} \\
& =r_{i} a_{i} .
\end{aligned}
$$

Therefore, by (4.4), we have $r_{i}=r \mathbf{J}_{i j} h_{j}$ for some $r$, where $i=$ $1, \ldots, 2 n$.

Since $\mathcal{H}_{2}$ is also skew orthogonal to $\mathcal{H}_{1}$, we also have

$$
\begin{aligned}
0 & =\omega\left(h_{0} h_{k} \vec{\alpha}_{k}-h_{j} \mathbf{J}_{j k} \vec{h}_{k}-H \vec{\alpha}_{0}-h_{j} h_{l} \Gamma_{0 l}^{k} \mathbf{J}_{j k} \vec{\alpha}_{0}\right. \\
& \left.-h_{j} h_{l} \mathbf{J}_{j s} \Gamma_{k l}^{s} \vec{\alpha}_{k}, 2 H \vec{h}_{0}-h_{0} \vec{H}+r_{0} \vec{\alpha}_{0}+r \mathbf{J}_{i j} h_{j} \vec{\alpha}_{i}\right) \\
& =-2 H d h_{0}\left(h_{j} \mathbf{J}_{j k} \vec{h}_{k}+H \vec{\alpha}_{0}+h_{j} h_{l} \Gamma_{0 l}^{k} \mathbf{J}_{j k} \vec{\alpha}_{0}\right) \\
& -h_{0} d H\left(h_{0} h_{k} \vec{\alpha}_{k}-h_{j} \mathbf{J}_{j k} \vec{h}_{k}-h_{j} h_{l} \mathbf{J}_{j s} \Gamma_{k l}^{s} \vec{\alpha}_{k}\right)-r \mathbf{J}_{i j} h_{j} \alpha_{i}\left(h_{l} \mathbf{J}_{l k} \vec{h}_{k}\right) \\
& =-2 H h_{j} \mathbf{J}_{j k} d h_{0}\left(\vec{h}_{k}\right)+(2 H)^{2}+2 H h_{j} h_{l} \Gamma_{0 l}^{k} \mathbf{J}_{j k} \\
& +4 h_{0}^{2} H+h_{0} h_{j} h_{l} \mathbf{J}_{j k} d h_{l}\left(\vec{h}_{k}\right)-h_{0} h_{i} h_{j} h_{l} \mathbf{J}_{j s} \Gamma_{i l}^{s}+2 r H \\
& =2 r H .
\end{aligned}
$$

Therefore $r=0$. Finally, since $2 H \vec{h}_{0}-h_{0} \vec{H}+r_{0} \vec{\alpha}_{0}$ is in $\mathcal{H}_{1}$, it follows from the structural equation that

$$
\begin{aligned}
0 & =\omega\left(\left[\vec{H}, 2 H \vec{h}_{0}-h_{0} \vec{H}+r_{0} \vec{\alpha}_{0}\right], 2 h_{0} h_{k} \vec{\alpha}_{k}-h_{j} \mathbf{J}_{j k} \vec{h}_{k}-2 H \vec{\alpha}_{0}\right) \\
& =r_{0} \omega\left(\left[\vec{H}, \vec{\alpha}_{0}\right], 2 h_{0} h_{k} \vec{\alpha}_{k}-h_{j} \mathbf{J}_{j k} \vec{h}_{k}-2 H \vec{\alpha}_{0}\right) .
\end{aligned}
$$

Hence, $r_{0}=0$ and this gives $\mathcal{H}_{1}$. 


\section{Curvatures of sub-Riemannian geodesic flows}

In this section, we will focus on the computation of the curvature $R^{i j}(0)$, where the Jacobi curve is given by the sub-Riemannian geodesic flow. For this, let $\mathcal{R}^{i j}: \mathcal{V}_{i} \rightarrow \mathcal{V}_{j}$ be the operator for which the matrix representation with respect to bases $E^{i}(0)$ and $E^{j}(0)$ of $\mathcal{V}_{i}$ and $\mathcal{V}_{j}$, respectively, is given by $R^{i j}(0)$. More precisely,

$$
\mathcal{R}^{i j}\left(E_{k}^{i}(0)\right)=\sum_{l} R_{k l}^{i j}(0) E_{l}^{j}(0),
$$

where $R_{k l}^{i j}(0)$ is the $k l$-th entry of $R^{i j}(0)$.

Theorem 5.1. Assume that the manifold is Sasakian. Then, under the identifications of Theorem 4.2. $\mathcal{R}$ is given by

(1) $\mathcal{R}(v)=0$ for all $v$ in $\mathcal{V}_{1}$,

(2) $\mathcal{R}(v)_{\mathcal{V}_{2}}=\left(R m\left(\boldsymbol{J} p^{h}, p^{h}\right) p^{h}\right)_{\mathcal{V}_{2}}+\left(\frac{1}{4}\left|p^{h}\right|^{2}+p\left(v_{0}\right)^{2}\right) \boldsymbol{J} p^{h}$ $=\left(R m^{*}\left(\boldsymbol{J} p^{h}, p^{h}\right) p^{h}\right) \mathcal{V}_{2}+p\left(v_{0}\right)^{2} \boldsymbol{J}^{h}$ for all $v$ in $\mathcal{V}_{2}$,

(3) $\mathcal{R}(v)_{\mathcal{V}_{3}}=\left(R m\left(\boldsymbol{J} p^{h}, p^{h}\right) p^{h}\right)_{\mathcal{V}_{3}}=\left(R m^{*}\left(\boldsymbol{J} p^{h}, p^{h}\right) p^{h}\right)_{\mathcal{V}_{3}}$ for all $v$ in $\mathcal{V}_{2}$

(4) $\mathcal{R}(v)_{\mathcal{V}_{1}}=0$ for all $v$ in $\mathcal{V}_{3}$

(5) $\mathcal{R}(v)_{\mathcal{V}_{2}}=\left(R m\left(v^{h}, p^{h}\right) p^{h}\right)_{\mathcal{V}_{2}}=\left(R m^{*}\left(\boldsymbol{J}_{p^{h}}, p^{h}\right) p^{h}\right) \mathcal{V}_{2}$ for all $v$ in $\mathcal{V}_{3}$

(6) $\mathcal{R}\left(p^{h}+p\left(v_{0}\right) v_{0}\right)=0$,

(7) $\mathcal{R}(v)_{\mathcal{V}_{3}}=\left(R m\left(v^{h}, p^{h}\right) p^{h}\right) \mathcal{V}_{3}+\frac{1}{4} p\left(v_{0}\right)^{2} v^{h}=\left(R m^{*}\left(v^{h}, p^{h}\right) p^{h}\right) \mathcal{V}_{3}$ $+\frac{1}{4} p\left(v_{0}\right)^{2} v^{h}$ for all $v$ in $\mathcal{V}_{3}$ satisfying $\left\langle v^{h}, p^{h}\right\rangle=0$.

Proof. Let $\Lambda_{\mathcal{V}_{i} \mathcal{H}_{j}}: \mathcal{V}_{i} \rightarrow \mathcal{H}_{j}$ be the operator defined by

$$
\Lambda_{\mathcal{V}_{i} \mathcal{H}_{j}}(V)=[\vec{H}, V]_{\mathcal{H}_{j}},
$$

where $V$ is a section in $\mathcal{V}_{i}$ and the subscript $\mathcal{H}_{j}$ denotes the $\mathcal{H}_{j^{-}}$ component of the vector.

It follows from (2.1) that $\Lambda_{\mathcal{V}_{i} \mathcal{H}_{j}}$ is tensorial and so well-defined. We also define operators $\Lambda_{\mathcal{V}_{i} \mathcal{V}_{j}}, \Lambda_{\mathcal{H}_{i} \mathcal{V}_{j}}$, and $\Lambda_{\mathcal{H}_{i} \mathcal{H}_{j}}$ in a similar way. By (2.1), we have

Lemma 5.2. The following relations hold.

(1) $\mathcal{R}^{11}=\Lambda_{\mathcal{H}_{1} \mathcal{V}_{1}} \circ \Lambda_{\mathcal{H}_{2} \mathcal{H}_{1}} \circ \Lambda_{\mathcal{V}_{2} \mathcal{H}_{2}} \circ \Lambda_{\mathcal{V}_{1} \mathcal{V}_{2}}$

(2) $\mathcal{R}^{13}=\Lambda_{\mathcal{H}_{1} \mathcal{V}_{3}} \circ \Lambda_{\mathcal{H}_{2} \mathcal{H}_{1}} \circ \Lambda_{\mathcal{V}_{2} \mathcal{H}_{2}} \circ \Lambda_{\mathcal{V}_{1} \mathcal{V}_{2}}$

(3) $\mathcal{R}^{22}=-\Lambda_{\mathcal{H}_{2} \mathcal{V}_{2}} \circ \Lambda_{\mathcal{V}_{2} \mathcal{H}_{2}}$,

(4) $\mathcal{R}^{23}=-\Lambda_{\mathcal{H}_{2} \mathcal{V}_{3}} \circ \Lambda_{\mathcal{V}_{2} \mathcal{H}_{2}}$,

(5) $\mathcal{R}^{31}=-\Lambda_{\mathcal{H}_{3} \mathcal{V}_{1}} \circ \Lambda_{\mathcal{V}_{3} \mathcal{H}_{3}}$,

(6) $\mathcal{R}^{32}=-\Lambda_{\mathcal{H}_{3} \mathcal{V}_{2}} \circ \Lambda_{\mathcal{V}_{3} \mathcal{H}_{3}}$

(7) $\mathcal{R}^{33}=-\Lambda_{\mathcal{H}_{3} \mathcal{V}_{3}} \circ \Lambda_{\mathcal{V}_{3} \mathcal{H}_{3}}$. 
Clearly, $\Lambda_{\mathcal{H}_{1} \mathcal{V}_{1}} \equiv 0$ and $\Lambda_{\mathcal{H}_{1} \mathcal{V}_{3}} \equiv 0$. For the rest, we need a lemma for which the proof is given in the appendix.

Lemma 5.3. The following holds at $x$

(1) $\left[\vec{h}_{k}, \vec{h}_{i}\right]=\boldsymbol{J}_{k i} \vec{h}_{0}+\sum_{a} b_{k i}^{a} \vec{\alpha}_{a}$

(2) $\sum_{k \neq 0} h_{k} b_{k i}^{0}=\sum_{k, s \neq 0} h_{k} h_{s} v_{k}\left(\Gamma_{0 i}^{s}\right)$ if $k, i \neq 0$,

(3) $\sum_{k \neq 0} h_{k} b_{k i}^{l}=-\sum_{s, k \neq 0} h_{s} h_{k}\left[v_{k} \Gamma_{i l}^{s}-v_{k} \Gamma_{l i}^{s}-v_{i} \Gamma_{k l}^{s}\right]$ if $k, i, l \neq 0$,

(4) $\left[\vec{H}, \vec{h}_{i}\right]=\sum_{k \neq 0} h_{k} \boldsymbol{J}_{k i} \vec{h}_{0}-\sum_{k \neq 0} h_{0} \boldsymbol{J}_{i k} \vec{h}_{k}+\sum_{k \neq 0, a} h_{k} b_{k i}^{a} \vec{\alpha}_{a}$.

Let $a_{i} \vec{h}_{i}+c_{a} \vec{\alpha}_{a}$ be a vector in $\mathcal{H}_{3}$. A computation shows that the followings hold at $x$.

$$
\begin{aligned}
& {\left[\vec{H}, a_{i} \vec{h}_{i}+c_{a} \vec{\alpha}_{a}\right]} \\
& =\left(\vec{H} a_{i}\right) \vec{h}_{i}+\left(\vec{H} c_{0}\right) \vec{\alpha}_{0}+\left(\vec{H} c_{i}\right) \vec{\alpha}_{i}+a_{i}\left[\vec{H}, \vec{h}_{i}\right]+c_{0}\left[\vec{H}, \vec{\alpha}_{0}\right]+c_{i}\left[\vec{H}, \vec{\alpha}_{i}\right] \\
& =\left(\vec{H} a_{i}\right) \vec{h}_{i}+a_{i} h_{j} h_{l}\left(v_{l} \Gamma_{0 j}^{i}\right) \vec{\alpha}_{0}+\left(\vec{H} c_{i}\right) \vec{\alpha}_{i}-h_{0} a_{i} \mathbf{J}_{i k} \vec{h}_{k} \\
& +a_{i} h_{k} h_{s}\left(v_{k} \Gamma_{0 i}^{s}\right) \vec{\alpha}_{0}+a_{i} h_{k} b_{k i}^{j} \vec{\alpha}_{j}+c_{k}\left(\vec{h}_{k}+h_{j} \mathbf{J}_{j k} \vec{\alpha}_{0}\right) \\
& =\left(\vec{H} a_{k}\right) \vec{h}_{k}-\frac{1}{2}\left(a_{j} \mathbf{J}_{j k} h_{0}+a_{0} h_{j} \mathbf{J}_{j k}\right) \vec{h}_{k}+\left(\vec{H} c_{i}\right) \vec{\alpha}_{i}+a_{i} h_{k} b_{k i}^{j} \vec{\alpha}_{j} .
\end{aligned}
$$

On the other hand, we have

$$
\frac{h_{0}}{2 H}\left(\vec{H} a_{k}-\frac{1}{2} a_{j} \mathbf{J}_{j k} h_{0}-\frac{1}{2} a_{0} h_{j} \mathbf{J}_{j k}\right) h_{k}=\frac{h_{0}}{2 H}\left(\vec{H} a_{k}\right) h_{k}
$$

and

$$
\begin{aligned}
& \frac{1}{2}\left(\vec{H} a_{i}-\frac{1}{2} a_{j} \mathbf{J}_{j i} h_{0}-\frac{1}{2} a_{0} h_{j} \mathbf{J}_{j i}\right) \mathbf{J}_{i k} h_{0}-\frac{1}{2} \frac{h_{0}}{2 H}\left(\vec{H} a_{i}\right) h_{i} h_{j} \mathbf{J}_{j k} \\
& =\frac{1}{2}\left(\vec{H} a_{i}\right) \mathbf{J}_{i k} h_{0}+\frac{1}{4} a_{k} h_{0}^{2}+\frac{1}{4} a_{0} h_{k} h_{0}-\frac{h_{0}}{4 H}\left(\vec{H} a_{i}\right) h_{i} h_{j} \mathbf{J}_{j k}
\end{aligned}
$$

at $x$.

Therefore,

$$
\begin{aligned}
& {\left[\vec{H}, a_{i} \vec{h}_{i}+c_{a} \vec{\alpha}_{a}\right] \mathcal{v}=-\frac{1}{2}\left(\vec{H} a_{i}\right) \mathbf{J}_{i k} h_{0} \vec{\alpha}_{k}-\frac{1}{4} a_{k} h_{0}^{2} \vec{\alpha}_{k}} \\
& -\frac{1}{4} a_{0} h_{k} h_{0} \vec{\alpha}_{k}+\frac{h_{0}}{4 H}\left(\vec{H} a_{i}\right) h_{i} h_{j} \mathbf{J}_{j k} \vec{\alpha}_{k}+\left(\vec{H} c_{k}\right) \vec{\alpha}_{k}+a_{i} h_{j} b_{j i}^{k} \vec{\alpha}_{k} .
\end{aligned}
$$


Another computation shows that

$$
\begin{aligned}
& \vec{H} c_{k}=\frac{1}{2}\left(\vec{H} a_{j}\right) \mathbf{J}_{j k} h_{0}-\frac{1}{2}\left(\vec{H} a_{0}\right) h_{j} \mathbf{J}_{j k}-\frac{1}{2} a_{0} h_{l} d h_{j}\left(\vec{h}_{l}\right) \mathbf{J}_{j k}+a_{i} h_{j} h_{l}\left(v_{l} \Gamma_{k j}^{i}\right) \\
& =\frac{1}{2}\left(\vec{H} a_{j}\right) \mathbf{J}_{j k} h_{0}-\frac{h_{0}}{4 H}\left(\vec{H} a_{l}\right) h_{l} h_{j} \mathbf{J}_{j k} \\
& -\frac{h_{0}}{4 H} a_{l} h_{s} d h_{l}\left(\vec{h}_{s}\right) h_{j} \mathbf{J}_{j k}-\frac{1}{2} a_{0} h_{l} d h_{j}\left(\vec{h}_{l}\right) \mathbf{J}_{j k}+a_{i} h_{j} h_{l}\left(v_{l} \Gamma_{k j}^{i}\right) \\
& =\frac{1}{2}\left(\vec{H} a_{j}\right) \mathbf{J}_{j k} h_{0}-\frac{h_{0}}{4 H}\left(\vec{H} a_{l}\right) h_{l} h_{j} \mathbf{J}_{j k}+\frac{1}{2} a_{0} h_{0} h_{k}+a_{i} h_{j} h_{l}\left(v_{l} \Gamma_{k j}^{i}\right)
\end{aligned}
$$

Hence,

$$
\begin{aligned}
& {\left[\vec{H}, a_{i} \vec{h}_{i}+c_{a} \vec{\alpha}_{a}\right] \mathcal{V}=-\frac{1}{2}\left(\vec{H} a_{i}\right) \mathbf{J}_{i k} h_{0} \vec{\alpha}_{k}-\frac{1}{4} a_{k} h_{0}^{2} \vec{\alpha}_{k}-\frac{1}{4} a_{0} h_{k} h_{0} \vec{\alpha}_{k}} \\
& +\frac{h_{0}}{4 H}\left(\vec{H} a_{i}\right) h_{i} h_{j} \mathbf{J}_{j k} \vec{\alpha}_{k}+\frac{1}{2}\left(\vec{H} a_{j}\right) \mathbf{J}_{j k} h_{0} \vec{\alpha}_{k}-\frac{h_{0}}{4 H}\left(\vec{H} a_{l}\right) h_{l} h_{j} \mathbf{J}_{j k} \vec{\alpha}_{k} \\
& +\frac{1}{2} a_{0} h_{0} h_{k} \vec{\alpha}_{k}+a_{i} h_{j} h_{l}\left(v_{l} \Gamma_{k j}^{i}\right) \vec{\alpha}_{k}+a_{i} h_{j} b_{j i}^{k} \vec{\alpha}_{k} \\
& =-\frac{1}{4} a_{k} h_{0}^{2} \vec{\alpha}_{k}+\frac{1}{4} a_{0} h_{k} h_{0} \vec{\alpha}_{k}-a_{i} h_{s} h_{l}\left(v_{l} \Gamma_{i k}^{s}-v_{i} \Gamma_{l k}^{s}\right) \vec{\alpha}_{k} \\
& =-\frac{1}{4} h_{0}\left(a_{k} h_{0}-a_{0} h_{k}\right) \vec{\alpha}_{k}-a_{i} h_{s} h_{l} \operatorname{Rm}_{i l s k} \vec{\alpha}_{k} .
\end{aligned}
$$

where $\operatorname{Rm}_{i j k s}=\left\langle\operatorname{Rm}\left(v_{i}, v_{j}\right) v_{k}, v_{s}\right\rangle$.

This finishes the proof of the last four assertions. Let

$$
h_{j} \mathbf{J}_{j k} \vec{h}_{k}-h_{0} h_{k} \vec{\alpha}_{k}+H \vec{\alpha}_{0}+h_{j} h_{l} \Gamma_{0 l}^{k} \mathbf{J}_{j k} \vec{\alpha}_{0}+h_{j} h_{l} \mathbf{J}_{j s} \Gamma_{k l}^{s} \vec{\alpha}_{k}
$$

be a section of the bundle $\mathcal{H}_{2}$. Then

$$
\begin{aligned}
& {\left[\vec{H}, h_{j} \mathbf{J}_{j k} \vec{h}_{k}-h_{0} h_{k} \vec{\alpha}_{k}+H \vec{\alpha}_{0}+h_{j} h_{l} \Gamma_{0 l}^{k} \mathbf{J}_{j k} \vec{\alpha}_{0}+h_{j} h_{l} \mathbf{J}_{j s} \Gamma_{k l}^{s} \vec{\alpha}_{k}\right]} \\
& =h_{i} d h_{j}\left(\vec{h}_{i}\right) \mathbf{J}_{j k} \vec{h}_{k}-h_{0} h_{i} d h_{k}\left(\vec{h}_{i}\right) \vec{\alpha}_{k}+H\left[\vec{H}, \vec{\alpha}_{0}\right]+h_{j} \mathbf{J}_{j k}\left[\vec{H}, \vec{h}_{k}\right] \\
& -h_{0} h_{k}\left[\vec{H}, \vec{\alpha}_{k}\right]+h_{j} h_{l} h_{i}\left(v_{i} \Gamma_{0 l}^{k}\right) \mathbf{J}_{j k} \vec{\alpha}_{0}+h_{j} h_{l} h_{i}\left(v_{i} \Gamma_{k l}^{s}\right) \mathbf{J}_{j s} \vec{\alpha}_{k} \\
& =-2 h_{0} \vec{H}-h_{0}^{2} h_{i} \mathbf{J}_{i k} \vec{\alpha}_{k}-H h_{j} \mathbf{J}_{j k} \vec{\alpha}_{k}+h_{j} \mathbf{J}_{j k}\left(h_{i} \mathbf{J}_{i k} \vec{h}_{0}-h_{0} \mathbf{J}_{k i} \vec{h}_{i}\right) \\
& +h_{j} h_{i} \mathbf{J}_{j k} b_{i k}^{a} \vec{\alpha}_{a}+h_{j} h_{l} h_{i}\left(v_{i} \Gamma_{0 l}^{k}\right) \mathbf{J}_{j k} \vec{\alpha}_{0}+h_{j} h_{l} h_{i}\left(v_{i} \Gamma_{k l}^{s}\right) \mathbf{J}_{j s} \vec{\alpha}_{k} \\
& =2 H \vec{h}_{0}-h_{0} \vec{H}-h_{0}^{2} h_{i} \mathbf{J}_{i k} \vec{\alpha}_{k}-H h_{j} \mathbf{J}_{j k} \vec{\alpha}_{k} \\
& +h_{j} h_{i} \mathbf{J}_{j k} b_{i k}^{a} \vec{\alpha}_{a}+h_{j} h_{l} h_{i}\left(v_{i} \Gamma_{0 l}^{k}\right) \mathbf{J}_{j k} \vec{\alpha}_{0}+h_{j} h_{l} h_{i}\left(v_{i} \Gamma_{k l}^{s}\right) \mathbf{J}_{j s} \vec{\alpha}_{k} .
\end{aligned}
$$


It follows that

$$
\begin{aligned}
& {\left[\vec{H}, h_{j} \mathbf{J}_{j k} \vec{h}_{k}-h_{0} h_{k} \vec{\alpha}_{k}+H \vec{\alpha}_{0}+h_{j} h_{l} \Gamma_{0 l}^{k} \mathbf{J}_{j k} \vec{\alpha}_{0}+h_{j} h_{l} \mathbf{J}_{j s} \Gamma_{k l}^{s} \vec{\alpha}_{k}\right]_{\mathcal{V}}} \\
& =-h_{0}^{2} h_{i} \mathbf{J}_{i k} \vec{\alpha}_{k}-H h_{j} \mathbf{J}_{j k} \vec{\alpha}_{k} \\
& +h_{j} h_{i} \mathbf{J}_{j k} b_{i k}^{a} \vec{\alpha}_{a}+h_{j} h_{l} h_{i}\left(v_{i} \Gamma_{0 l}^{k}\right) \mathbf{J}_{j k} \vec{\alpha}_{0}+h_{j} h_{l} h_{i}\left(v_{i} \Gamma_{k l}^{s}\right) \mathbf{J}_{j s} \vec{\alpha}_{k} \\
& =-h_{0}^{2} h_{i} \mathbf{J}_{i k} \vec{\alpha}_{k}-H h_{j} \mathbf{J}_{j k} \vec{\alpha}_{k}+h_{j} h_{i} h_{s} \mathbf{J}_{j k}\left(v_{i} \Gamma_{0 k}^{s}\right) \vec{\alpha}_{0} \\
& +h_{j} h_{l} h_{i}\left(v_{i} \Gamma_{0 l}^{k}\right) \mathbf{J}_{j k} \vec{\alpha}_{0}-h_{s} h_{j} h_{k} \mathbf{J}_{j i}\left(v_{k} \Gamma_{i l}^{s}-v_{k} \Gamma_{l i}^{s}-v_{i} \Gamma_{k l}^{s}+v_{k} \Gamma_{l i}^{s}\right) \vec{\alpha}_{l} \\
& =-h_{0}^{2} h_{i} \mathbf{J}_{i k} \vec{\alpha}_{k}-H h_{j} \mathbf{J}_{j k} \vec{\alpha}_{k}-h_{s} h_{j} h_{k} \mathbf{J}_{j i}\left(v_{k} \Gamma_{i l}^{s}-v_{i} \Gamma_{k l}^{s}\right) \vec{\alpha}_{l} \\
& =-\left(h_{0}^{2}+\frac{1}{2} H\right) h_{i} \mathbf{J}_{i k} \vec{\alpha}_{k}-h_{j} h_{k} h_{s} \mathbf{J}_{j i} \operatorname{Rm}_{k i l s} \vec{\alpha}_{l} .
\end{aligned}
$$

\section{Conjugate time estimates and Bonnet-MyeR's type THEOREM}

In this section, we give estimates for the first conjugate time under certain curvature lower bound. Let $\psi_{t}: T_{x}^{*} M \rightarrow M$ be the map defined by $\psi_{t}(x, p)=\pi\left(e^{t \vec{H}}(x, p)\right)$, where $\pi: T^{*} M \rightarrow M$ is the projection. Let us fix a covector $(x, p)$. The first conjugate time is the smallest $t_{0}>0$ such that the linear map $\left(d \psi_{t_{0}}\right)_{(x, p)}$ is not bijective. The curve $t \mapsto \psi_{t}(x, p)$ is no longer minimizing if $t>t_{0}$ (see [2]).

Theorem 6.1. Assume that the Tanaka-Webster curvature Rm* of the Sasakian manifold satisfies

(1) $\left\langle R m^{*}\left(\boldsymbol{J}_{p^{h}}, p^{h}\right) p^{h}, \boldsymbol{J}_{p^{h}}\right\rangle \geq k_{1}\left|p^{h}\right|^{4}$,

(2) $\sum_{i=1}^{2 n-2}\left\langle R m^{*}\left(w_{i}, p^{h}\right) p^{h}, w_{i}\right\rangle \geq(2 n-1) k_{2}\left|p^{h}\right|^{2}$,

for some non-negative constants $k_{1}$ and $k_{2}$, where $w_{1}, \ldots, w_{2 n-2}$ is an orthonormal frame of $\left\{p^{h}, \boldsymbol{J}_{p^{h}}, v_{0}\right\}^{\perp}$. Then the first conjugate time of the geodesic $t \mapsto \psi_{t}(x, p)$ is less than or equal to $\frac{2 \pi}{\sqrt{p\left(v_{0}\right)^{2}+k_{1}\left|p^{h}\right|^{2}}}$ and $\frac{2 \pi}{\sqrt{p\left(v_{0}\right)^{2}+4 k_{2}\left|p^{h}\right|^{2}}}$.

Moreover, if

(1) $\left\langle R m^{*}\left(\boldsymbol{J}^{h}, p^{h}\right) p^{h}, \boldsymbol{J} p^{h}\right\rangle=k_{1}\left|p^{h}\right|^{4}$,

(2) $\sum_{i=1}^{2 n-2}\left\langle R m^{*}\left(w_{i}, p^{h}\right) p^{h}, w_{i}\right\rangle=(2 n-1) k_{2}\left|p^{h}\right|^{2}$.

Then the first conjugate time of the geodesic $t \mapsto \psi_{t}(x, p)$ is equal to the minimum of $\frac{2 \pi}{\sqrt{p\left(v_{0}\right)^{2}+k_{1}\left|p^{h}\right|^{2}}}$ and $\frac{2 \pi}{\sqrt{p\left(v_{0}\right)^{2}+4 k_{2}\left|p^{h}\right|^{2}}}$. 
Proof. Let $E(t)=\left(E^{1}(t), E^{2}(t), E^{3}(t)\right), F(t)=\left(F^{1}(t), F^{2}(t), F^{3}(t)\right)$ be a canonical frame of the Jacobi curve $\mathfrak{J}_{(x, p)}(t)$. Let $A(t)$ and $B(t)$ be matrices defined by

$$
E(0)=A(t) E(t)+B(t) F(t) .
$$

On the other hand, if we differentiate the equation (6.1) with respect to $t$, then

$$
\begin{aligned}
0 & =\dot{A}(t) E(t)+A(t) \dot{E}(t)+\dot{B}(t) F(t)+B(t) \dot{F}(t) \\
& =\dot{A}(t) E(t)+A(t) C_{1} E(t)+A(t) C_{2} F(t) \\
& +\dot{B}(t) F(t)-B(t) R(t) E(t)-B(t) C_{1}^{T} F(t) .
\end{aligned}
$$

It follows that

$$
\begin{aligned}
& \dot{A}(t)+A(t) C_{1}-B(t) R(t)=0 \\
& \dot{B}(t)+A(t) C_{2}-B(t) C_{1}^{T}=0
\end{aligned}
$$

with initial conditions $B(0)=0$ and $A(0)=I$.

If we set $S(t)=B(t)^{-1} A(t)$, then $S(t)$ satisfies the following Riccati equation

$$
\dot{S}(t)-S(t) C_{2} S(t)+C_{1}^{T} S(t)+S(t) C_{1}-R(t)=0 .
$$

Let us choose $E_{2 n-1}^{3}(0)=p^{h}+p\left(v_{0}\right) v$ and let

$$
S(t)=\left(\begin{array}{ccc}
S_{1}(t) & S_{2}(t) & S_{3}(t) \\
S_{2}(t)^{T} & S_{4}(t) & S_{5}(t) \\
S_{3}(t)^{T} & S_{5}(t)^{T} & S_{6}(t)
\end{array}\right),
$$

where $S_{1}(t)$ is a $2 \times 2$ matrix and $S_{6}(t)$ is $1 \times 1$. Then

$$
\begin{aligned}
& \dot{S}_{1}(t)-S_{1}(t) \tilde{C}_{2} S_{1}(t)-S_{2}(t) S_{2}(t)^{T} \\
& \quad-S_{3}(t) S_{3}(t)^{T}+\tilde{C}_{1}^{T} S_{1}(t)+S_{1}(t) \tilde{C}_{1}-\tilde{R}^{1}(t)=0 \\
& \dot{S}_{4}(t)-S_{4}(t)^{2}-S_{5}(t) S_{5}(t)^{T}-S_{2}(t)^{T} \tilde{C}_{2} S_{2}(t)-\tilde{R}^{2}(t)=0 \\
& \dot{S}_{6}(t)-S_{6}(t)^{2}-S_{5}(t)^{T} S_{5}(t)-S_{3}(t)^{T} \tilde{C}_{2} S_{3}(t)=0
\end{aligned}
$$

where $\tilde{C}_{1}=\left(\begin{array}{ll}0 & 1 \\ 0 & 0\end{array}\right), \tilde{C}_{2}=\left(\begin{array}{cc}0 & 0 \\ 0 & 1\end{array}\right)$, and $\tilde{R}^{1}(t)=\left(\begin{array}{cc}0 & 0 \\ 0 & R^{22}(t)\end{array}\right)$. $\tilde{R}^{2}(t)$ is the $(2 n-2) \times(2 n-2)$ matrix with $i j$-th entry equal to $R_{i j}^{33}(t)$.

Note that $U(t)=S(t)^{-1}$ also satisfies $U(0)=0$ and the Riccati equation

$$
\dot{U}(t)+C_{2}-U(t) C_{1}^{T}-C_{1} U(t)+U(t) R(t) U(t)=0 .
$$


This gives

$$
U(t)=-t C_{2}-\frac{t^{2}}{2}\left(C_{1}+C_{1}^{T}\right)-\frac{t^{3}}{6}\left(C_{1} C_{1}^{T}+C_{2} R(0) C_{2}\right)+O\left(t^{4}\right) .
$$

By using this expansion and $S(t) U(t)=I$, we obtain

$$
\begin{gathered}
S_{1}(t)=\left(\begin{array}{cc}
-\frac{12}{t^{3}}+O\left(1 / t^{2}\right) & \frac{6}{t^{2}}+O(1 / t) \\
\frac{6}{t^{2}}+O(1 / t) & -\frac{4}{t}+O(1)
\end{array}\right), \\
\operatorname{tr}\left(S_{4}(t)\right)=-\frac{2 n-2}{t}+O(1), \quad S_{6}(t)=-\frac{1}{t}+O(1) .
\end{gathered}
$$

(For instance, one can take the dot product of the first row

$$
s(t)=\left(S_{1,1}(t), \ldots, S_{1,2 n+1}(t)\right)
$$

of $S(t)$ with the third, fourth, .., $2 n$-th columns of $U(t)$. This gives the order of the dominating terms of $\left(S_{1,3}(t), \ldots, S_{1,2 n+1}(t)\right)$ in terms of that of $S_{1,2}(t)$. By taking the dot product of $s(t)$ with the first and second column of $U(t)$, we obtain the leading order terms of $S_{1,1}(t)$ and $S_{1,2}(t)$. Similar procedure works for other entries of $S(t)$.)

By applying the comparison principle of Riccati equations in [12] to $S(t)$, we have $S_{1}(t) \geq \Gamma_{1}(t)$, where $\Gamma_{1}(t)$ is a solution of the following Riccati equation

$$
\dot{\Gamma}_{1}(t)-\Gamma_{1}(t) \tilde{C}_{2} \Gamma_{1}(t)+\tilde{C}_{1}^{T} \Gamma_{1}(t)+\Gamma_{1}(t) \tilde{C}_{1}-K_{1}=0
$$

with the initial condition $\lim _{t \rightarrow 0} \Gamma_{1}^{-1}(t)=0$. (Of course, one needs to apply the comparison principle to $S(t)$ and $\Gamma(t+\epsilon)$ and let $\epsilon$ to zero as usual). Here $K_{1}=\left(\begin{array}{cc}0 & 0 \\ 0 & \mathfrak{k}_{1}\end{array}\right)$ and $\mathfrak{k}_{1}=p\left(v_{0}\right)^{2}+k_{1}\left|p^{h}\right|^{2}$. Thus

$$
\begin{aligned}
\operatorname{tr}\left(\tilde{C}_{2} S_{1}(t)\right) & \geq \operatorname{tr}\left(\tilde{C}_{2} \Gamma_{1}(t)\right) \\
& =\frac{\sqrt{\mathfrak{k}_{1}}\left(\sqrt{\mathfrak{k}_{1}} t \cos \left(\sqrt{\mathfrak{k}_{1}} t\right)-\sin \left(\sqrt{\mathfrak{k}_{1}} t\right)\right)}{\left(2-2 \cos \left(\sqrt{\mathfrak{k}_{1}} t\right)-\sqrt{\mathfrak{k}_{1}} t \sin \left(\sqrt{\mathfrak{k}_{1}} t\right)\right)} .
\end{aligned}
$$

For the term $S_{4}(t)$, we can take the trace and obtain

$$
\frac{d}{d t} \operatorname{tr}\left(S_{4}(t)\right) \geq \frac{1}{2 n-2} \operatorname{tr}\left(S_{4}(t)\right)^{2}+(2 n-2) \mathfrak{k}_{2},
$$

where $\mathfrak{k}_{2}=\frac{1}{4} p\left(v_{0}\right)^{2}+k_{2}\left|p^{h}\right|^{2}$.

Now applying the comparison principle in [12] again we have

$$
\left.\operatorname{tr}\left(S_{4}(t)\right) \geq-\sqrt{\mathfrak{k}_{2}}(2 n-2) \cot \left(\sqrt{\mathfrak{k}_{2}} t\right)\right) .
$$

Finally, for the term $S_{6}(t)$, we have

$$
\dot{S}_{6}(t) \geq S_{6}(t)^{2} .
$$


which implies

$$
S_{6}(t) \geq-\frac{1}{t}
$$

By combining this with (6.5) and (6.6), we obtain

$$
\begin{aligned}
\operatorname{tr}\left(C_{2} S(t)\right) & \geq-\sqrt{\mathfrak{k}_{2}}(2 n-2) \cot \left(\sqrt{\mathfrak{k}_{2}} t\right)-\frac{1}{t} \\
& +\frac{\sqrt{\mathfrak{k}_{1}}\left(\sqrt{\mathfrak{k}_{1}} t \cos \left(\sqrt{\mathfrak{k}_{1}} t\right)-\sin \left(\sqrt{\mathfrak{k}_{1}} t\right)\right)}{\left(2-2 \cos \left(\sqrt{\mathfrak{k}_{1}} t\right)-\sqrt{\mathfrak{k}_{1}} t \sin \left(\sqrt{\mathfrak{k}_{1}} t\right)\right)} .
\end{aligned}
$$

Therefore,

$$
\begin{aligned}
& \frac{d}{d t} \log |\operatorname{det} B(t)|=\operatorname{tr}\left(C_{1}^{T}-S(t) C_{2}\right)=-\operatorname{tr}\left(C_{2} S(t)\right) \\
& \leq \sqrt{\mathfrak{k}_{2}}(2 n-2) \cot \left(\sqrt{\mathfrak{k}_{2}} t\right)+\frac{1}{t}-\frac{\sqrt{\mathfrak{k}_{1}}\left(\sqrt{\mathfrak{k}_{1}} t \cos \left(\sqrt{\mathfrak{k}_{1}} t\right)-\sin \left(\sqrt{\mathfrak{k}_{1}} t\right)\right)}{\left(2-2 \cos \left(\sqrt{\mathfrak{k}_{1}} t\right)-\sqrt{\mathfrak{k}_{1}} t \sin \left(\sqrt{\mathfrak{k}_{1}} t\right)\right)}
\end{aligned}
$$

and hence

$$
|\operatorname{det} B(t)| \leq C a(t)
$$

where $C=\lim _{t_{0} \rightarrow 0} \frac{\left|\operatorname{det} B\left(t_{0}\right)\right|}{a\left(t_{0}\right)}$ and

$$
a(t)=t \sin ^{2 n-2}\left(\sqrt{\mathfrak{k}_{2}} t\right)\left(2-2 \cos \left(\sqrt{\mathfrak{k}_{1}} t\right)-\sqrt{\mathfrak{k}_{1}} t \sin \left(\sqrt{\mathfrak{k}_{1}} t\right)\right) .
$$

Using (6.2) and the definition of determinant, we see that $B(t)=$ $-C_{2} t+\frac{1}{2}\left(C_{1}-C_{1}^{T}\right) t^{2}+\frac{1}{6}\left(C_{2} R(0) C_{2}+C_{1} C_{1}^{T}\right) t^{3}+O\left(t^{4}\right)$ and $|\operatorname{det} B(t)|=$ $\frac{1}{12} t^{2 n+3}+O\left(t^{2 n+4}\right)$.

Therefore,

$$
|\operatorname{det} B(t)| \leq \frac{t \sin ^{2 n-2}\left(\sqrt{\mathfrak{k}_{2}} t\right)\left(2-2 \cos \left(\sqrt{\mathfrak{k}_{1}} t\right)-\sqrt{\mathfrak{k}_{1}} t \sin \left(\sqrt{\mathfrak{k}_{1}} t\right)\right)}{\mathfrak{k}_{1}^{2} \mathfrak{k}_{2}^{2 n-2}}
$$

The first assertion follows. Let $S^{k_{1}, k_{2}}(t)$ be a solution of (6.3) with $R(t)$ replaced by

$$
R^{k_{1}, k_{2}}=\left(\begin{array}{cccc}
0 & 0 & 0 & 0 \\
0 & \mathfrak{k}_{1} & 0 & 0 \\
0 & 0 & \mathfrak{k}_{2} I_{2 n-2} & 0 \\
0 & 0 & 0 & 0
\end{array}\right)
$$

with the initial condition $\lim _{t \rightarrow 0}\left(S_{t}^{k_{1}, k_{2}}\right)^{-1}=0$, where $\mathfrak{k}_{1}=p\left(v_{0}\right)^{2}$ and $\mathfrak{k}_{2}=\frac{1}{4} p\left(v_{0}\right)^{2}$. 
A calculation similar to that of Theorem 6.1 shows that

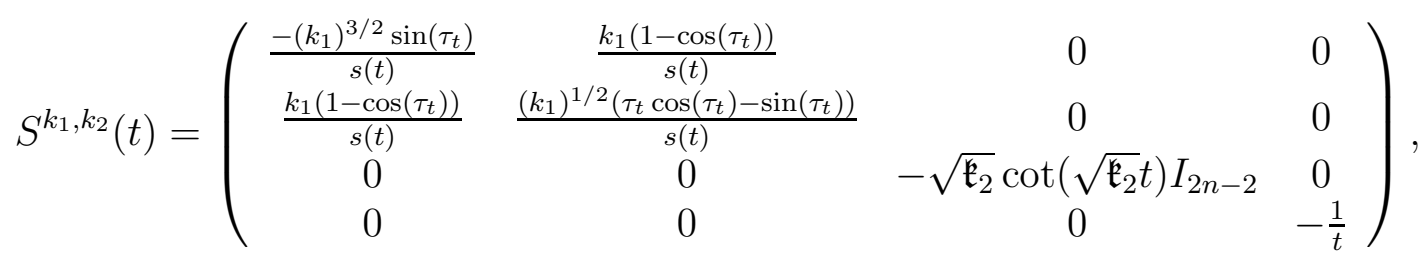

where $\tau_{t}=\sqrt{\mathfrak{k}_{1}} t$ and $s(t)=2-2 \cos \left(\tau_{t}\right)-\tau_{t} \sin \left(\tau_{t}\right)$.

The rest follows as the proof of the previous assertion (with all inequalities replaced by equalities).

\section{Model Cases}

In this section, we discuss two examples, the Heisenberg group and the complex Hopf fibration which are relevant to the later sections. First, we consider a family of Sasakian manifolds $\left(M, \mathbf{J}, v_{0}, \alpha_{0}, g=\right.$ $\langle\cdot, \cdot\rangle)$ for which the quotient of $M$ by the flow of $v_{0}$ is a manifold $B$. Since $\mathcal{L}_{v_{0}} \mathbf{J}=0$ and $\mathcal{L}_{v_{0}} g=0$, they descend to a complex structure $\mathbf{J}_{B}$ and a Riemannian metric $g_{B}$ on $B$. Moreover, by Theorem 3.2 , they form a Kähler manifold. Moreover, the Tanaka-Webster curvature Rm* on $M$ and the Riemann curvature tensor $\mathrm{Rm}^{B}$ of $B$ are related by

Lemma 7.1. The curvature tensors $R m^{*}$ and $R m^{B}$ are related by

$$
\operatorname{Rm}^{*}(\bar{X}, \bar{Y}) \bar{Z}=\overline{R m^{B}(X, Y) Z}
$$

where $\bar{X}$ denotes the vector orthogonal to $v_{0}$ which project to the vector $X$.

Proof. Since $M \rightarrow B$ is a Riemannian submersion, we have (see [11])

$$
\nabla_{\bar{X}}^{*} \bar{Y}=\left(\nabla_{\bar{X}} \bar{Y}\right)^{h}=\overline{\nabla_{X} Y}
$$

Since $\bar{Z}$ projects to $Z$, we also have

$$
\nabla_{v_{0}}^{*} \bar{Z}=\left(\nabla_{v_{0}} \bar{Z}\right)^{h}+\frac{1}{2} \mathbf{J} \bar{Z}=\left(\nabla_{\bar{Z}} v_{0}\right)^{h}+\frac{1}{2} \mathbf{J} \bar{Z}=0 .
$$

Therefore,

$$
\begin{aligned}
& \operatorname{Rm}^{*}(\bar{X}, \bar{Y}) \bar{Z}=\nabla_{\bar{X}}^{*} \nabla_{\bar{Y}}^{*} \bar{Z}-\nabla_{\bar{Y}}^{*} \nabla_{\bar{X}}^{*} \bar{Z}-\nabla_{[\bar{X}, \bar{Y}]}^{*} \bar{Z} \\
& =\overline{\nabla_{X} \nabla_{Y} Z}-\overline{\nabla_{Y} \nabla_{X} Z}-\overline{\nabla_{[X, Y]} Z}-\alpha_{0}([\bar{X}, \bar{Y}]) \nabla_{v_{0}}^{*} \bar{Z} \\
& =\overline{\mathrm{Rm}^{B}(X, Y) Z} \text {. }
\end{aligned}
$$


The first example is the Heisenberg group. In this case the manifold $M$ is the Euclidean space $\mathbb{R}^{2 n+1}$. If we fix a coordinate system $\left(x_{1}, \ldots, x_{n}, y_{1}, \ldots, y_{n}, z\right)$, then the 1 -form $\alpha_{0}$ and the vector field $v_{0}$, are given, respectively, by

$$
\alpha_{0}=d z-\frac{1}{2} \sum_{i=1}^{n} x_{i} d y_{i}+\frac{1}{2} \sum_{i=1}^{n} y_{i} d x_{i} \quad \text { and } \quad v_{0}=\partial_{z} .
$$

The Riemannian metric is the one for which the frame

$$
X_{i}=\partial_{x_{i}}-\frac{1}{2} y_{i} \partial_{z}, \quad Y_{i}=\partial_{y_{i}}+\frac{1}{2} x_{i} \partial_{z}, \quad \partial_{z}
$$

is orthonormal. The tensor $\mathbf{J}$ is defined by

$$
\mathbf{J}\left(X_{i}\right)=Y_{i}, \quad \mathbf{J}\left(Y_{i}\right)=-X_{i}, \quad \mathbf{J}\left(\partial_{z}\right)=0 .
$$

The quotient $B$ is $\mathbb{C}^{n}$ equipped with the standard complex structure and Euclidean inner product.

Let $(x, p)$ be a covector with $\left|p^{h}\right|=1$. Assume that $t \mapsto \psi(x, t \epsilon p)$ is length minimizing between its endpoints for some $\epsilon>0$. Then, we define the cut time of $(x, p)$ to be the largest such $\epsilon$. The following is well-known. We give the proof for completeness.

Theorem 7.2. On the Heisenberg group equipped with the above subRiemannian structure, the cut time coincides with the first conjugate time.

Proof. Let $P_{X_{i}}=p_{x_{i}}-\frac{1}{2} y_{i} p_{z}$ and $P_{Y_{i}}=p_{y_{i}}+\frac{1}{2} x_{i} p_{z}$. A computation as in [10] shows that

$$
\begin{aligned}
& P_{j}(t):=P_{X_{j}}(t)+i P_{Y_{j}}(t)=P_{j}(0) e^{i t p_{z}} \\
& w_{j}(t):=x_{j}(t)+i y_{j}(t)=w_{j}(0)-\frac{i P_{j}(0)}{p_{z}}\left(e^{i t p_{z}}-1\right), \\
& z(t):=z(0)+\frac{1}{2} \sum_{k=1}^{n} \int_{0}^{t} \operatorname{Im}\left(\bar{w}_{k}(s) \dot{w}_{k}(s)\right) d s .
\end{aligned}
$$

If $(w, z)$ and $(\tilde{w}, \tilde{z})$ are unit speed geodesics with the same length $L$ and end-points, then

$$
\frac{\tilde{P}_{j}(0)}{\tilde{p}_{z}}\left(e^{i L \tilde{p}_{z}}-1\right)=\frac{P_{j}(0)}{p_{z}}\left(e^{i L p_{z}}-1\right) .
$$

By taking the norms, it follows that

$$
\frac{1-\cos \left(L \tilde{p}_{z}\right)}{\tilde{p}_{z}^{2}}=\frac{1-\cos \left(L p_{z}\right)}{p_{z}^{2}} .
$$


Using $w_{j}(0)=\tilde{w}_{j}(0)$ and $w_{j}(L)=\tilde{w}_{j}(L)$, we also have

$$
\frac{e^{i \tilde{\theta}}}{\tilde{p}_{z}}\left(e^{i L \tilde{p}_{z}}-1\right)=\frac{e^{i \theta}}{p_{z}}\left(e^{i L p_{z}}-1\right),
$$

where $P_{j}(0)=e^{i \theta}$ and $\tilde{P}_{j}(0)=e^{i \tilde{\theta}}$. Therefore,

$$
\begin{aligned}
& \frac{\cos \left(\theta+L p_{z}\right)-\cos (\theta)}{p_{z}}=\frac{\cos \left(\tilde{\theta}+L \tilde{p}_{z}\right)-\cos (\tilde{\theta})}{\tilde{p}_{z}} \\
& \frac{\sin \left(\theta+L p_{z}\right)-\sin (\theta)}{p_{z}}=\frac{\sin \left(\tilde{\theta}+L \tilde{p}_{z}\right)-\sin (\tilde{\theta})}{\tilde{p}_{z}}
\end{aligned}
$$

Finally, since $z(L)=\tilde{z}(L)$, a computation together with the above implies that

$$
\frac{L \tilde{p}_{z}-\sin \left(L \tilde{p}_{z}\right)}{\tilde{p}_{z}^{2}}=\frac{L p_{z}-\sin \left(L p_{z}\right)}{p_{z}^{2}} .
$$

By investigating the graph of $\frac{1-\cos (x)}{x^{2}}$ and $\frac{x-\sin (x)}{x^{2}}$, we have $p_{z}=\tilde{p}_{z}$. Therefore, if $L<\frac{2 \pi}{p_{z}}$, then $P_{j}(0)=\tilde{P}_{j}(0)$ and the two geodesics coincide. Hence, the result follows from Theorem 6.1.

The second example is the complex Hopf fibration. We follow the discussion in [4]. In this case, the manifold is given by the sphere $S^{2 n+1}=\left\{z \in \mathbb{C}^{n+1}|| z \mid=1\right\}$. The 1 -form $\alpha_{0}$ and the vector field $v_{0}$ are given, respectively, by

$$
\alpha_{0}=\frac{1}{2} \sum_{i=1}^{n}\left(x_{i} d y_{i}-y_{i} d x_{i}\right)
$$

and

$$
v_{0}=2 \sum_{i=1}^{n}\left(-y_{i} \partial_{x_{i}}+x_{i} \partial_{y_{i}}\right)
$$

where $z_{j}=x_{j}+i y_{j}$.

The tangent space of $S^{2 n+1}$ is the direct sum of ker $\alpha_{0}$ and $\mathbb{R} v_{0}$. The Riemannian metric is defined in such a way that $v_{0}$ has length one, $v_{0}$ is orthogonal to ker $\alpha_{0}$, and the restriction of the metric to ker $\alpha_{0}$ coincides with the Euclidean one. The (1,1)-tensor $\mathbf{J}$ is defined analogously by the conditions $\mathbf{J} v_{0}=0$ and the restriction of $\mathbf{J}$ to ker $\alpha_{0}$ coincides with the standard complex structure on $\mathbb{C}^{n}$. The base manifold $B$ is the complex projective space $\mathbb{C P}^{n}$ and the induced Riemannian metric is given by the Fubini-Study metric. It follows from Lemma 7.1 that

$$
\left\langle\operatorname{Rm}^{*}(\mathbf{J} X, X) X, \mathbf{J} X\right\rangle=4 \text { and }\left\langle\operatorname{Rm}^{*}(v, X) X, v\right\rangle=1
$$

for all $v$ in the orthogonal complement of $\{X, J X\}$. 
Theorem 7.3. On the complex Hopf fibration equipped with the above sub-Riemannian structure, the cut time coincides with the first conjugate time.

Proof. The sub-Riemannian geodesic flow is given by

$$
\left(a \cos (|v| t)+\frac{v}{|v|} \sin (|v| t)\right) e^{-i t\left\langle v_{0}, v\right\rangle},
$$

where $a$ is the initial point of the geodesic and $v$ is the initial (co)vector (see [10, 4]).

By the choice of the complex coordinate system, we can assume $a=(1,0, \ldots, 0)$. Let $v=\left(v_{1}, \ldots, v_{n}\right)$. Then the real part of $v_{1}$ equal 0 . Moreover, $v^{h}=\left(0, v_{2}, \ldots, v_{n}\right)$ is the horizontal part of $v$. Assume that $\left|v^{h}\right|=1$ and let $w$ be another such covector such that the corresponding geodesic has the same end point and the same length $L$ as that of $v$.

Under the above assumptions, we have

$$
|v|^{2}-\frac{1}{4}\left(\operatorname{Im}\left(v_{1}\right)\right)^{2}=1=|w|^{2}-\frac{1}{4}\left(\operatorname{Im}\left(w_{1}\right)\right)^{2}
$$

and

$$
\begin{aligned}
& \left(a \cos (\|v\| L)+\frac{v}{\|v\|} \sin (\|v\| L)\right) e^{-\frac{i L}{2} \operatorname{Im}\left(v_{1}\right)} \\
& =\left(a \cos (\|w\| L)+\frac{w}{\|w\|} \sin (\|w\| L)\right) e^{-\frac{i L}{2} \operatorname{Im}\left(w_{1}\right)} .
\end{aligned}
$$

It follows that

$$
\begin{aligned}
& \left(\cos (|v| L)+\frac{v_{1}}{|v|} \sin (|v| L)\right) e^{-\frac{i L}{2} \operatorname{Im}\left(v_{1}\right)} \\
& =\left(\cos (|w| L)+\frac{w_{1}}{|w|} \sin (|w| L)\right) e^{-\frac{i L}{2} \operatorname{Im}\left(w_{1}\right)}
\end{aligned}
$$

and

$$
\left(\frac{v_{i}}{|v|} \sin (|v| L)\right) e^{-\frac{i L}{2} \operatorname{Im}\left(v_{1}\right)}=\left(\frac{w_{i}}{|w|} \sin (|w| L)\right) e^{-\frac{i L}{2} \operatorname{Im}\left(w_{1}\right)} .
$$

for all $i \neq 1$.

By taking the norm of the second equation, we obtain

$$
\frac{\left|v_{i}\right|^{2}}{|v|^{2}} \sin ^{2}(|v| L)=\frac{\left|w_{i}\right|^{2}}{|w|^{2}} \sin ^{2}(|w| L)
$$

If we sum over $i \neq 1$, then we have

$$
\frac{\sin ^{2}(|v| L)}{|v|^{2}}=\frac{\sin ^{2}(|w| L)}{|w|^{2}}
$$


If both $|v|$ and $|w|$ are less than or equal to $\frac{\pi}{L}$, then $|v|=|w|$. It follows that $\operatorname{Im}\left(v_{1}\right)= \pm \operatorname{Im}\left(w_{1}\right)$.

If $\operatorname{Im}\left(v_{1}\right)=\operatorname{Im}\left(w_{1}\right)$, then either $v_{i}=w_{i}$ for all $i$ which implies that the two geodesics coincide or $\sin (L|v|)=0=\sin (L|w|)$. In this case $|v|=|w|=\frac{\pi}{L}$.

If $\operatorname{Im}\left(v_{1}\right)=-\operatorname{Im}\left(w_{1}\right)$, then

$$
\left(\cos (|v|)+\frac{v_{1}}{|v|} \sin (|v|)\right) e^{i L \operatorname{Im}\left(v_{1}\right)}=\left(\cos (|v|)-\frac{v_{1}}{|v|} \sin (|v|)\right) .
$$

It follows that

$$
\frac{\tan (|v|)}{|v|}=\frac{\tan (\operatorname{Im}(v) / 2)}{\operatorname{Im}(v) / 2}
$$

Since $|v|>\frac{1}{2} \operatorname{Im}(v)$, we have a contradiction. Therefore, the result from this and Theorem 6.1.

\section{Volume growth estimates}

In this section, we prove a volume growth estimate and the proof of Theorem 1.1 and 1.2. Let $\Omega$ be the set of points $(x, p)$ in the cotangent space $T_{x}^{*} M$ such that the curve $t \in[0,1] \mapsto \psi_{t}(x, p)$ is a length minimizing. Let

$$
\Sigma=\left\{p \in \Omega|| p^{h} \mid=1 \text { and } \epsilon p \in \Omega \text { for some } \epsilon>0\right\} .
$$

For each $p$ in $\Sigma$, we let $T(p)$ be the cut time which is the maximal time $T$ such that $t \in[0, T] \mapsto \psi_{t}(x, p)$ is length minimizing. Finally, let us denote the ball centered at $x$ of radius $R$ with respect to the sub-Riemannian distance by $B_{R}(x)$ and the Riemannian volume form by $\eta$.

Theorem 8.1. Assume that the Tanaka-Webster curvature Rm* of the Sasakian manifold satisfies

(1) $\left\langle R m^{*}\left(\boldsymbol{J} p^{h}, p^{h}\right) p^{h}, \boldsymbol{J}_{p^{h}}\right\rangle \geq k_{1}\left|p^{h}\right|^{4}$,

(2) $\sum_{i=1}^{2 n-2}\left\langle R m^{*}\left(w_{i}, p^{h}\right) p^{h}, w_{i}\right\rangle \geq(2 n-1) k_{2}\left|p^{h}\right|^{2}$,

for some constants $k_{1}$ and $k_{2}$, where $w_{1}, \ldots, w_{2 n-2}$ is an orthonormal frame of $\boldsymbol{s p a n}\left\{p^{h}, \boldsymbol{J} p^{h}, v_{0}\right\}^{\perp}$. Then

$$
\int_{B_{R}(x)} d \eta \leq \int_{0}^{\min \{T(p), R\}} \int_{\Sigma} k(r, z) d \mathfrak{m}(r, z)
$$

where $(r, z)$ denotes the cylindrical coordinates defined by $r=\left|p^{h}\right|$ and $z=p\left(v_{0}\right), \mathfrak{k}_{1}(r, z)=z^{2}+k_{1} r^{2}, \mathfrak{k}_{2}(r, z)=\frac{1}{4} z^{2}+k_{2} r^{2}$. The function $k$ is 
defined by

$$
k(r, z)=r^{2}\left[\frac{\sin ^{2 n-2}\left(\sqrt{\mathfrak{k}_{2}}\right)\left(2-2 \cos \left(\sqrt{\mathfrak{k}_{1}}\right)-\sqrt{\mathfrak{k}_{1}} \sin \left(\sqrt{\mathfrak{k}_{1}}\right)\right)}{\mathfrak{k}_{1}^{2} \mathfrak{k}_{2}^{2 n-2}}\right]
$$

if $\mathfrak{k}_{1} \geq 0$ and $\mathfrak{k}_{2} \geq 0$,

$$
k(r, z)=r^{2}\left[\frac{\sinh ^{2 n-2}\left(\sqrt{-\mathfrak{k}_{2}}\right)\left(2-2 \cos \left(\sqrt{\mathfrak{k}_{1}}\right)-\sqrt{\mathfrak{k}_{1}} \sin \left(\sqrt{\mathfrak{k}_{1}}\right)\right)}{\mathfrak{k}_{1}^{2} \mathfrak{k}_{2}^{2 n-2}}\right]
$$

if $\mathfrak{k}_{1} \geq 0$ and $\mathfrak{k}_{2} \leq 0$,

$$
k(r, z)=r^{2}\left[\frac{\sin ^{2 n-2}\left(\sqrt{\mathfrak{k}_{2}}\right)\left(2-2 \cosh \left(\sqrt{-\mathfrak{k}_{1}}\right)+\sqrt{-\mathfrak{k}_{1}} \sinh \left(\sqrt{-\mathfrak{k}_{1}}\right)\right)}{\mathfrak{k}_{1}^{2} \mathfrak{k}_{2}^{2 n-2}}\right]
$$

if $\mathfrak{k}_{1} \leq 0$ and $\mathfrak{k}_{2} \geq 0$,

$k(r, z)=r^{2}\left[\frac{\sinh ^{2 n-2}\left(\sqrt{-\mathfrak{k}_{2}}\right)\left(2-2 \cosh \left(\sqrt{-\mathfrak{k}_{1}}\right)+\sqrt{-\mathfrak{k}_{1}} \sinh \left(\sqrt{-\mathfrak{k}_{1}}\right)\right)}{\mathfrak{k}_{1}^{2} \mathfrak{k}_{2}^{2 n-2}}\right]$

if $\mathfrak{k}_{1} \leq 0$ and $\mathfrak{k}_{2} \leq 0$.

Proof. We use the same notations as in the proof of Theorem 6.1.

Let $\rho_{t}: T_{x}^{*} M \rightarrow \mathbb{R}$ be the function defined by $\psi_{t}^{*} \eta=\rho_{t} \mathfrak{m}$. It follows from Theorem 4.3 that

$$
\rho_{t}=\left|p^{h}\right|^{2}|\operatorname{det} B(t)| \text {. }
$$

Next, we replace the matrix $R(t)$ in (6.2) by $R^{k_{1}, k_{2}}$ and denote the solutions by $A^{k_{1}, k_{2}}(t)$ and $B^{k_{1}, k_{2}}(t)$. Then

$$
\frac{\frac{d}{d t} \operatorname{det} B(t)}{\operatorname{det} B(t)}=-\operatorname{tr}\left(S(t) C_{2}\right) \leq-\operatorname{tr}\left(S^{k_{1}, k_{2}}(t) C_{2}\right)=\frac{\frac{d}{d t} \operatorname{det} B^{k_{1}, k_{2}}(t)}{\operatorname{det} B^{k_{1}, k_{2}}(t)} .
$$

It follows that $\frac{\operatorname{det} B(t)}{\operatorname{det} B^{k_{1}, k_{2}(t)}}$ is non-increasing.

It follows that from the proof of Theorem 6.1 that

$$
\begin{aligned}
& \int_{B_{R}(x)} d \eta=\int_{\Sigma} \int_{0}^{\min \{T(p), R\}} \rho_{t} d \mathfrak{m} \\
& \leq \int_{\Omega_{R}}\left|p^{h}\right|^{2}\left[\frac{\sin ^{2 n-2}\left(\sqrt{\mathfrak{k}_{2}}\right)\left(2-2 \cos \left(\sqrt{\mathfrak{k}_{1}}\right)-\sqrt{\mathfrak{k}_{1}} \sin \left(\sqrt{\mathfrak{k}_{1}}\right)\right)}{\mathfrak{k}_{1}^{2} \mathfrak{k}_{2}^{2 n-2}}\right] d \mathfrak{m}(p) .
\end{aligned}
$$

Proof of Theorem 1.1 and 1.2. By the proof of Theorem 6.1]and Theorem 7.3 , the volume of sub-Riemannian ball of radius $R$ in the Complex Hopf fibration is given by

$$
\int_{\Omega_{R}}\left|p^{h}\right|^{2}\left[\frac{\sin ^{2 n-2}\left(\sqrt{\mathfrak{k}_{2}}\right)\left(2-2 \cos \left(\sqrt{\mathfrak{k}_{1}}\right)-\sqrt{\mathfrak{k}_{1}} \sin \left(\sqrt{\mathfrak{k}_{1}}\right)\right)}{\mathfrak{k}_{1}^{2} \mathfrak{k}_{2}^{2 n-2}}\right] d \mathfrak{m}(p) .
$$

Therefore, the result follows from 8.1. 


\section{LAPLACIAN COMPARISON THEOREM}

In this section, we define a version of Hessian following [1 and prove Theorem 1.3 .

Let $f: M \rightarrow \mathbb{R}$ be a smooth function. The graph $G$ of the differential $d f$ defines a sub-manifold of the manifold $T^{*} M$. Let $v$ be a tangent vector in $T_{x} M$. Then there is a vector $X$ in the tangent space of $G$ at $d f_{x}$ such that $\pi_{*}(X)=v$, where $\pi: T^{*} M \rightarrow M$ is the projection. The sub-Riemannian Hessian Hess $f$ at $x$ is defined by Hess $f(v)=X_{\mathcal{V}}$. Recall that $X_{\mathcal{V}}$ is the component of $X$ in $\mathcal{V}$ with respect to the splitting $T T^{*} M=\mathcal{V} \oplus \mathcal{H}$.

Lemma 9.1. Under the identification in Theorem 4.2, the sub-Riemannian Hessian is given by

(1) Hess $f(v)=\nabla_{v} \nabla f$ if $v$ is contained in the orthogonal complement of $\left\{\nabla f^{h}, \boldsymbol{J} \nabla f, v_{0}\right\}$

(2) Hess $f\left(\nabla f^{h}\right)=\nabla_{\nabla f^{h}} \nabla f-\frac{1}{2}\left\langle\nabla f, v_{0}\right\rangle \boldsymbol{J} \nabla f^{h}$,

(3) Hess $f(\boldsymbol{J} \nabla f)=\nabla_{\boldsymbol{J} \nabla f} \nabla f-\frac{1}{2}\left\langle\nabla f, v_{0}\right\rangle \nabla f^{h}+\frac{1}{2}\left|\nabla f^{h}\right|^{2} v_{0}$,

(4) Hess $f(v)=\nabla_{v} \nabla f+\frac{|\nabla f|^{2}}{2} \boldsymbol{J} \nabla f$ if $v=\left|\nabla f^{h}\right|^{2} v_{0}-\left(v_{0} f\right) \nabla f^{h}$.

Proof. Let $\left\{v_{0}, \ldots, v_{2 n}\right\}$ be a frame defined as in Lemma 3.7 around a point $x$. Since $\pi_{*}\left(\vec{h}_{i}\right)=v_{i}$, we have

$$
(d f)_{*}\left(k_{a} v_{a}\right)=k_{a} \vec{h}_{a}+\bar{k}_{a} \vec{\alpha}_{a} .
$$

It follows that

$$
\begin{aligned}
\bar{k}_{c}+k_{a} d h_{a}\left(\vec{h}_{c}\right) & =\omega\left(\vec{h}_{c},(d f)_{*}\left(k_{a} v_{a}\right)\right)=-d h_{c}\left((d f)_{*}\left(k_{a} v_{a}\right)\right) \\
=-k_{a}\left(v_{a} v_{c} f\right) & =-k_{a}\left\langle\nabla_{v_{a}} \nabla f, v_{c}\right\rangle-k_{a}\left\langle\nabla f, \nabla_{v_{a}} v_{c}\right\rangle .
\end{aligned}
$$

Therefore, we have the following at $x$.

$$
\begin{aligned}
\bar{k}_{i} & =-k_{a}\left\langle\nabla_{v_{a}} \nabla f, v_{i}\right\rangle-k_{a}\left\langle\nabla f, \nabla_{v_{a}} v_{i}\right\rangle-k_{a} d h_{a}\left(\vec{h}_{i}\right) \\
& =-k_{a}\left\langle\nabla_{v_{a}} \nabla f, v_{i}\right\rangle-\frac{k_{j}}{2} \mathbf{J}_{j i} v_{0} f-k_{0} d h_{0}\left(\vec{h}_{i}\right)-k_{j} d h_{j}\left(\vec{h}_{i}\right) \\
& =-k_{a}\left\langle\nabla_{v_{a}} \nabla f, v_{i}\right\rangle+\frac{k_{j}}{2} \mathbf{J}_{j i} v_{0} f+\frac{k_{0}}{2} \mathbf{J}_{i k} v_{k} f
\end{aligned}
$$

and

$$
\begin{aligned}
& \bar{k}_{0}=-k_{a}\left\langle\nabla_{v_{a}} \nabla f, v_{0}\right\rangle-k_{i}\left\langle\nabla f, \nabla_{v_{i}} v_{0}\right\rangle-k_{i} d h_{i}\left(\vec{h}_{0}\right) \\
& =-k_{a}\left\langle\nabla_{v_{a}} \nabla f, v_{0}\right\rangle+\frac{k_{i}}{2}\left\langle\mathbf{J} v_{i}, \nabla f^{h}\right\rangle-\frac{1}{2} k_{i} \mathbf{J}_{i j} h_{j}=-k_{a}\left\langle\nabla_{v_{a}} \nabla f, v_{0}\right\rangle .
\end{aligned}
$$

Hence, if $v:=k_{a} v_{a}$ is contained in $\pi_{*} \mathcal{H}_{3}$, then

$$
\left((d f)_{*}\left(k_{i} v_{i}\right)\right)_{\mathcal{V}}=-\left(\frac{1}{2} k_{j} \mathbf{J}_{j i} v_{0} f-\frac{\left(v_{0} f\right)\left(v_{s} f\right) k_{s}}{2\left|\nabla f^{h}\right|^{2}}\left(v_{j} f\right) \mathbf{J}_{j i}\right) \vec{\alpha}_{i}+\bar{k}_{a} \vec{\alpha}_{a} .
$$


If $v$ is contained in $\pi_{*} \mathcal{H}_{3}$ and the orthogonal complement of $\nabla f^{h}$, then

$$
\left((d f)_{*}\left(k_{i} v_{i}\right)\right)_{\mathcal{V}}=-\left\langle\nabla_{k_{i} v_{i}} \nabla f, v_{a}\right\rangle \vec{\alpha}_{a}
$$

If $v=\nabla f^{h}$, then

$$
\left((d f)_{*}\left(\nabla f^{h}\right)\right)_{\mathcal{V}}=-\left\langle\nabla_{\nabla f^{h}} \nabla f, v_{a}\right\rangle \vec{\alpha}_{a}+\frac{1}{2}\left\langle\mathbf{J} \nabla f^{h}, v_{i}\right\rangle\left\langle\nabla f, v_{0}\right\rangle \vec{\alpha}_{i} .
$$

If $v=\mathbf{J} \nabla f^{h}$, then

$$
\begin{aligned}
\left((d f)_{*}(\mathbf{J} \nabla f)\right)_{\mathcal{V}} & =\left[\left(v_{j} f\right) \mathbf{J}_{j i} \vec{h}_{i}\right]_{\mathcal{V}}-\left\langle\nabla_{\mathbf{J} \nabla f} \nabla f, v_{0}\right\rangle \vec{\alpha}_{0} \\
& -\left\langle\nabla_{\mathbf{J} \nabla f} \nabla f, v_{i}\right\rangle \vec{\alpha}_{i}-\frac{v_{i} f}{2}\left(v_{0} f\right) \vec{\alpha}_{i} \\
& =-\left\langle\nabla_{\mathbf{J} \nabla f} \nabla f, v_{a}\right\rangle \vec{\alpha}_{a}+\frac{v_{i} f}{2}\left(v_{0} f\right) \vec{\alpha}_{i}-\frac{1}{2}\left|\nabla f^{h}\right|^{2} \vec{\alpha}_{0} .
\end{aligned}
$$

Finally, if $v=\left|\nabla f^{h}\right|^{2} v_{0}-\left(v_{0} f\right) \nabla f^{h}$, then we have

$$
\left((d f)_{*}(v)\right)_{\mathcal{V}}=-\left\langle\nabla_{v} \nabla f, v_{a}\right\rangle \vec{\alpha}_{a}-\frac{|\nabla f|^{2}}{2}\left\langle\mathbf{J} \nabla f, v_{i}\right\rangle \vec{\alpha}_{i}
$$

Proof of Theorem 1.3. Let $f(x)=-\frac{1}{2} d^{2}\left(x, x_{0}\right)$. Then the curve $t \in$ $[0,1] \mapsto \pi e^{t \vec{H}}\left(d f_{x}\right)$ is the geodesic which starts from $x$ and ends at $x_{0}$. Let $E(t)=\left(E^{1}(t), E^{2}(t), E^{3}(t)\right), F(t)=\left(F^{1}(t), F^{2}(t), F^{3}(t)\right)$ be a canonical frame of the Jacobi curve $\mathfrak{J}_{\left(x, d f_{x}\right)}(t)$. Let

$$
\mathcal{E}=\left(\mathcal{E}^{1}, \mathcal{E}^{2}, \mathcal{E}_{1}^{3}, \ldots, \mathcal{E}_{2 n-1}^{3}\right)^{T}, \mathcal{F}=\left(\mathcal{F}^{1}, \mathcal{F}^{2}, \mathcal{F}_{1}^{3}, \ldots, \mathcal{F}_{2 n-1}^{3}\right)^{T}
$$

be a symplectic basis of $T_{\left(x_{0}, p\right)} T^{*} M$ such that $\mathcal{E}^{i}$ is contained in $\mathcal{V}_{i}$ and $\mathcal{F}^{i}$ is contained in $\mathcal{H}_{i}$, where $\left(x_{0}, p\right)=e^{1 \cdot \vec{H}}\left(d f_{x}\right)$. Let

$$
v=\left(v^{1}, v^{2}, v_{1}^{3}, \ldots, v_{2 n-1}^{3}\right)^{T}
$$

be a basis of $T_{x} M$ such that $e_{*}^{t \vec{H}}\left(d f_{x}\right)_{*}(v)=\mathcal{E}$. Let $A(t)$ and $B(t)$ be matrices such that

$$
\left(d f_{x}\right)_{*}(v)=A(t) E(t)+B(t) F(t) .
$$

By construction, we have $B(1)=0$. We can also pick $E(t)$ such that $A(1)=I$.

By the definition of Hess $f$, we also have

$$
\text { Hess } f\left(B(0) \pi_{*} F(0)\right)=\text { Hess } f(v)=A(0) E(0) .
$$

Therefore, if we let $S(t)=B(t)^{-1} A(t)$, then

$$
\text { Hess } f\left(\pi_{*} F(0)\right)=S(0) E(0) \text {. }
$$


A computation as in the proof of Theorem 6.1 shows that

$$
\dot{S}(t)-S(t) C_{2} S(t)+C_{1}^{T} S(t)+S(t) C_{1}-R(t)=0 .
$$

Therefore, by applying similar computation as in the proof of Theorem 6.1 to $S(1-t)$, we obtain estimates for $S(0)$. Since $\Delta_{H} f(x)=$ $\operatorname{tr}\left(C_{2} S(0)\right)$, the result follows.

\section{Appendix I}

In this section, we give the proof of various known results in Section 3 .

Proof of Lemma 3.1. Since the almost contact manifold is normal, we have

$$
0=[\mathbf{J}, \mathbf{J}]\left(v, v_{0}\right)+d \alpha_{0}\left(v, v_{0}\right)=\mathbf{J}^{2}\left[v, v_{0}\right]-\mathbf{J}\left[\mathbf{J} v, v_{0}\right]=\mathbf{J} \mathcal{L}_{v_{0}}(\mathbf{J}) v .
$$

It follows that $\mathcal{L}_{v_{0}}(\mathbf{J})=0$.

Since the metric is associated to the almost contact structure,

$$
\begin{aligned}
0 & =\mathcal{L}_{v_{0}} \alpha_{0}(v)=\mathcal{L}_{v_{0}}\left(\left\langle v_{0}, v\right\rangle\right)-\alpha_{0}\left(\left[v_{0}, v\right]\right) \\
& =\left\langle\nabla_{v_{0}} v_{0}, v\right\rangle+\left\langle v_{0}, \nabla_{v_{0}} v\right\rangle-\left\langle v_{0}, \nabla_{v_{0}} v\right\rangle+\left\langle v_{0}, \nabla_{v} v_{0}\right\rangle \\
& =\left\langle\nabla_{v_{0}} v_{0}, v\right\rangle .
\end{aligned}
$$

Since the metric is associated to the almost contact structure and $\mathcal{L}_{v_{0}}(\mathbf{J})=0$, we also have

$$
\mathcal{L}_{v_{0}} g(v, \mathbf{J} w)=\left(\mathcal{L}_{v_{o}} d \alpha_{0}\right)(v, w)=0 .
$$

Therefore, $\mathcal{L}_{v_{0}} g=0$ as claimed.

By Lemma 3.7, we have

$$
\left\langle\mathbf{J}\left(v_{j}\right), v_{i}\right\rangle=\mathbf{J}_{j i}=2 \Gamma_{j i}^{0}=-2\left\langle\nabla_{v_{j}} v_{0}, v_{i}\right\rangle .
$$

Therefore, $\mathbf{J}=-2 \nabla v_{0}$.

Proof of Lemma 3.2. Let $v_{0}, v_{1}, \ldots, v_{2 n}$ be a local frame defined by Lemma 3.6. Then

$$
\begin{aligned}
0 & =\mathcal{L}_{v_{0}}(\mathbf{J})\left(v_{i}\right)=\left[v_{0}, \mathbf{J} v_{i}\right]-\mathbf{J}\left[v_{0}, v_{i}\right] \\
& =\nabla_{v_{0}}\left(\mathbf{J} v_{i}\right)-\nabla_{\mathbf{J} v_{i}}\left(v_{0}\right)-\mathbf{J} \nabla_{v_{0}} v_{i}+\mathbf{J} \nabla_{v_{i}} v_{0} \\
& =\left(\nabla_{v_{0}} \mathbf{J}\right) v_{i}-\nabla_{\mathbf{J} v_{i}}\left(v_{0}\right)+\mathbf{J} \nabla_{v_{i}} v_{0} \\
& =\left(\nabla_{v_{0}} \mathbf{J}\right) v_{i}+\frac{1}{2} \mathbf{J}^{2} v_{i}-\frac{1}{2} \mathbf{J}^{2} v_{i}=\left(\nabla_{v_{0}} \mathbf{J}\right) v_{i}
\end{aligned}
$$

Since $\mathbf{J} v_{0}=0$,

$$
\left(\nabla_{v_{i}} \mathbf{J}\right) v_{0}=-\mathbf{J} \nabla_{v_{i}} v_{0}=\frac{1}{2} \mathbf{J} \mathbf{J} v_{i}=-\frac{1}{2} v_{i} .
$$


Since $\nabla_{v_{0}} v_{0}=0$, we also have $\left(\nabla_{v_{0}} \mathbf{J}\right) v_{0}=-\mathbf{J}\left(\nabla_{v_{0}} v_{0}\right)=0$.

Finally, we need to show $\left(\nabla_{v_{i}} \mathbf{J}\right) v_{j}=\frac{1}{2} \delta_{i j} v_{0}$. First, by the properties of the frame $v_{1}, \ldots, v_{n}$, we have

$$
\left\langle\left(\nabla_{v_{i}} \mathbf{J}\right) v_{j}, v_{0}\right\rangle=-\left\langle\mathbf{J} v_{j}, \nabla_{v_{i}} v_{0}\right\rangle=\frac{1}{2}\left\langle\mathbf{J} v_{j}, \mathbf{J} v_{i}\right\rangle=\frac{1}{2} \delta_{i j}
$$

at $x$

By normality and properties of the frame $v_{1}, \ldots, v_{2 n}$, we have

$$
0=\left(\nabla_{\mathbf{J} v_{i}} \mathbf{J}\right) v_{j}-\left(\nabla_{\mathbf{J} v_{j}} \mathbf{J}\right) v_{i}+\mathbf{J}\left(\nabla_{v_{j}} \mathbf{J}\right) v_{i}-\mathbf{J}\left(\nabla_{v_{i}} \mathbf{J}\right) v_{j}+d \alpha_{0}\left(v_{i}, v_{j}\right) v_{0}
$$

It follows from Lemma 3.7 that

$$
\begin{aligned}
0 & =\left\langle\left(\nabla_{\mathbf{J} v_{i}} \mathbf{J}\right) v_{j}, v_{k}\right\rangle-\left\langle\left(\nabla_{\mathbf{J} v_{j}} \mathbf{J}\right) v_{i}, v_{k}\right\rangle+\left\langle\mathbf{J}\left(\nabla_{v_{j}} \mathbf{J}\right) v_{i}, v_{k}\right\rangle-\left\langle\mathbf{J}\left(\nabla_{v_{i}} \mathbf{J}\right) v_{j}, v_{k}\right\rangle \\
& =-\left\langle\left(\nabla_{v_{k}} \mathbf{J}\right) \mathbf{J} v_{i}, v_{j}\right\rangle-\left\langle\left(\nabla_{v_{j}} \mathbf{J}\right) v_{k}, \mathbf{J} v_{i}\right\rangle+\left\langle\left(\nabla_{v_{k}} \mathbf{J}\right) \mathbf{J} v_{j}, v_{i}\right\rangle \\
& +\left\langle\left(\nabla_{v_{i}} \mathbf{J}\right) v_{k}, \mathbf{J} v_{j}\right\rangle+\left\langle\mathbf{J}\left(\nabla_{v_{j}} \mathbf{J}\right) v_{i}, v_{k}\right\rangle-\left\langle\mathbf{J}\left(\nabla_{v_{i}} \mathbf{J}\right) v_{j}, v_{k}\right\rangle \\
& =-\left\langle\left(\nabla_{v_{k}} \mathbf{J}\right) \mathbf{J} v_{i}, v_{j}\right\rangle+\left\langle\left(\nabla_{v_{j}} \mathbf{J}\right) \mathbf{J} v_{i}, v_{k}\right\rangle+\left\langle\mathbf{J}\left(\nabla_{v_{k}} \mathbf{J}\right) v_{i}, v_{j}\right\rangle \\
& -\left\langle\left(\nabla_{v_{i}} \mathbf{J}\right) \mathbf{J} v_{j}, v_{k}\right\rangle+\left\langle\mathbf{J}\left(\nabla_{v_{j}} \mathbf{J}\right) v_{i}, v_{k}\right\rangle-\left\langle\mathbf{J}\left(\nabla_{v_{i}} \mathbf{J}\right) v_{j}, v_{k}\right\rangle .
\end{aligned}
$$

Since $\mathbf{J}^{2} v_{j}=-v_{j}$, we also have $\left\langle\left(\nabla_{v_{i}} \mathbf{J}\right) \mathbf{J} v_{j}, v_{k}\right\rangle=-\left\langle\mathbf{J}\left(\nabla_{v_{i}} \mathbf{J}\right) v_{j}, v_{k}\right\rangle$. Therefore, the above equation simplifies to

$$
0=-2\left\langle\left(\nabla_{v_{k}} \mathbf{J}\right) v_{i}, \mathbf{J} v_{j}\right\rangle \text {. }
$$

Proof of 3.3. Since the manifold is Sasakian, we have

$$
\begin{aligned}
\operatorname{Rm}(X, Y) v_{0} & =\nabla_{X} \nabla_{Y} v_{0}-\nabla_{Y} \nabla_{X} v_{0}-\nabla_{[X, Y]} v_{0} \\
& =\frac{1}{2}\left(-\nabla_{X}(\mathbf{J}(Y))+\nabla_{Y}(\mathbf{J}(X))+\mathbf{J}[X, Y]\right) \\
& =\frac{1}{2}\left(-\nabla_{X} \mathbf{J}(Y)+\nabla_{Y} \mathbf{J}(X)\right) \\
& =\frac{1}{4} \alpha_{0}(Y) X-\frac{1}{4} \alpha_{0}(X) Y .
\end{aligned}
$$

Proof of 3.4. Let $\nabla^{*}$ be the Tanaka connection defined by

$$
\nabla_{X}^{*} Y=\nabla_{X} Y+\alpha_{0}(X) \mathbf{J} Y-\alpha_{0}(Y) \nabla_{X} v_{0}+\nabla_{X} \alpha_{0}(Y) v_{0}
$$

Assume that $X$ and $Y$ are horizontal. Then

$$
\nabla_{X}^{*} Y=\nabla_{X} Y-\left\langle\nabla_{X} Y, v_{0}\right\rangle v_{0} \text {. }
$$

Therefore,

$$
\begin{aligned}
& \nabla_{X}^{*} \nabla_{Y}^{*} Z=\nabla_{X}\left(\nabla_{Y} Z-\left\langle\nabla_{Y} Z, v_{0}\right\rangle v_{0}\right)-\left\langle\nabla_{X}\left(\nabla_{Y} Z-\left\langle\nabla_{Y} Z, v_{0}\right\rangle v_{0}\right), v_{0}\right\rangle v_{0} \\
& =\nabla_{X} \nabla_{Y} Z-\left\langle\nabla_{X} \nabla_{Y} Z, v_{0}\right\rangle v_{0}-\left\langle\nabla_{Y} Z, v_{0}\right\rangle \nabla_{X} v_{0}
\end{aligned}
$$


Let $\mathrm{Rm}^{*}$ be the curvature corresponding to $\nabla^{*}$. Assume that $X, Y, Z$ are horizontal. Then

$$
\begin{aligned}
& \operatorname{Rm}^{*}(X, Y) Z=\nabla_{X}^{*} \nabla_{Y}^{*} Z-\nabla_{Y}^{*} \nabla_{X}^{*} Z-\nabla_{[X, Y]}^{*} Z \\
& =\nabla_{X} \nabla_{Y} Z-\left\langle\nabla_{X} \nabla_{Y} Z, v_{0}\right\rangle v_{0}-\left\langle\nabla_{Y} Z, v_{0}\right\rangle \nabla_{X} v_{0}-\nabla_{Y} \nabla_{X} Z \\
& +\left\langle\nabla_{Y} \nabla_{X} Z, v_{0}\right\rangle v_{0}+\left\langle\nabla_{X} Z, v_{0}\right\rangle \nabla_{Y} v_{0}-\nabla_{[X, Y]} Z+\left\langle\nabla_{[X, Y]} Z, v_{0}\right\rangle v_{0} \\
& =(\operatorname{Rm}(X, Y) Z)^{h}+\left\langle Z, \nabla_{Y} v_{0}\right\rangle \nabla_{X} v_{0}-\left\langle Z, \nabla_{X} v_{0}\right\rangle \nabla_{Y} v_{0} .
\end{aligned}
$$

\section{Appendix II}

This section is devoted to the proof of Lemma 4.4 and 5.3 .

Proof of Lemma 4.4. By the definition of $\vec{h}_{a}$, we have $\pi_{*}\left(\vec{h}_{a}\right)=v_{a}$. Therefore, the first relation follows. The second relation follows from $\pi_{*} \vec{\alpha}_{a}=0$.

Let $\theta$ be the tautological 1 -form defined by $\theta=p_{a} d x_{a}$. Note that $\theta\left(\vec{h}_{a}\right)=h_{a}$ and $\omega=d \theta$. The third relation follows from

$$
\begin{aligned}
d h_{b}\left(\vec{h}_{a}\right) & =d \theta\left(\vec{h}_{a}, \vec{h}_{b}\right) \\
& =\vec{h}_{a}\left(\theta\left(\vec{h}_{b}\right)\right)-\vec{h}_{b}\left(\theta\left(\vec{h}_{a}\right)\right)-\theta\left(\left[\vec{h}_{a}, \vec{h}_{b}\right]\right) \\
& =2 d h_{b}\left(\vec{h}_{a}\right)-\left(\Gamma_{a b}^{c}-\Gamma_{b a}^{c}\right) h_{c} .
\end{aligned}
$$

It is clear that $\left[\vec{\alpha}_{a}, \vec{h}_{b}\right]$ is vertical. The fourth relation follows from

$$
d h_{c}\left(\left[\vec{\alpha}_{a}, \vec{h}_{b}\right]\right)=\vec{\alpha}_{a}\left(d h_{c}\left(\vec{h}_{b}\right)\right)=\left(\Gamma_{b c}^{d}-\Gamma_{c b}^{d}\right) d h_{d}\left(\vec{\alpha}_{a}\right)=\Gamma_{c b}^{a}-\Gamma_{b c}^{a} .
$$

The fifth and sixth relations follow from the fourth one and $\vec{H}=h_{i} \vec{h}_{i}$. The seventh follows from the third.

The eighth relation follows from the fifth and the sixth. Indeed,

$$
\begin{aligned}
& {\left[\vec{H},\left[\vec{H}, \vec{\alpha}_{0}\right]\right]=-\vec{H}\left(h_{j} \mathbf{J}_{j k}\right) \vec{\alpha}_{k}-h_{j} \mathbf{J}_{j k}\left[\vec{H}, \vec{\alpha}_{k}\right]} \\
& =-h_{l} d h_{j}\left(\vec{h}_{l}\right) \mathbf{J}_{j k} \vec{\alpha}_{k}-h_{l} h_{j}\left(v_{l} \mathbf{J}_{j k}\right) \vec{\alpha}_{k}-h_{j} \mathbf{J}_{j k}\left(\vec{h}_{k}+h_{l}\left(\Gamma_{a l}^{k}-\Gamma_{l a}^{k}\right) \vec{\alpha}_{a}\right) \\
& =-h_{l} h_{j} \Gamma_{l s}^{j} \mathbf{J}_{s k} \vec{\alpha}_{k}+h_{0} h_{k} \vec{\alpha}_{k}-h_{l} h_{j}\left(\Gamma_{l j}^{s} \mathbf{J}_{s k}+\Gamma_{l k}^{s} \mathbf{J}_{j s}\right) \vec{\alpha}_{k} \\
& -h_{j} \mathbf{J}_{j k} \vec{h}_{k}-H \vec{\alpha}_{0}-h_{j} h_{l} \Gamma_{0 l}^{k} \mathbf{J}_{j k} \vec{\alpha}_{0}-h_{j} h_{l} \mathbf{J}_{j s}\left(\Gamma_{k l}^{s}-\Gamma_{l k}^{s}\right) \vec{\alpha}_{k} \\
& =h_{0} h_{k} \vec{\alpha}_{k}-h_{j} \mathbf{J}_{j k} \vec{h}_{k}-H \vec{\alpha}_{0}-h_{j} h_{l} \Gamma_{0 l}^{k} \mathbf{J}_{j k} \vec{\alpha}_{0}-h_{j} h_{l} \mathbf{J}_{j s} \Gamma_{k l}^{s} \vec{\alpha}_{k} .
\end{aligned}
$$

By the fifth relation, we have

$$
\left[\vec{H},\left[\vec{H}, \vec{\alpha}_{i}\right]\right]=\left[\vec{H}, \vec{h}_{i}\right]+h_{j}\left(\Gamma_{k j}^{i}-\Gamma_{j k}^{i}\right) \vec{h}_{k} \quad \text { (mod vertical) }
$$

when $i \neq 0$. 
Since $\pi_{*}\left[\vec{h}_{j}, \vec{h}_{k}\right]=\left[v_{j}, v_{k}\right]$, the above equation becomes $\left[\vec{H},\left[\vec{H}, \vec{\alpha}_{i}\right]\right]$

$=h_{l}\left(\Gamma_{l i}^{a}-\Gamma_{i l}^{a}\right) \vec{h}_{a}-h_{a}\left(\Gamma_{i k}^{a}-\Gamma_{k i}^{a}\right) \vec{h}_{k}+h_{l}\left(\Gamma_{k l}^{i}-\Gamma_{l k}^{i}\right) \vec{h}_{k} \quad(\bmod$ vertical $)$ $=2 h_{l} \Gamma_{l i}^{k} \vec{h}_{k}+h_{l} \mathbf{J}_{l i} \vec{h}_{0}-h_{0} \mathbf{J}_{i k} \vec{h}_{k} \quad$ (mod vertical).

Finally, by the sixth relation, we have $\left[\vec{H},\left[\vec{H},\left[\vec{H}, \vec{\alpha}_{0}\right]\right]\right]$ $=-2 \vec{H}\left(h_{j} \mathbf{J}_{j k}\right) \vec{h}_{k}-h_{j} \mathbf{J}_{j k}\left[\vec{H},\left[\vec{H}, \vec{\alpha}_{k}\right]\right](\bmod$ vertical $)$ $=-2 h_{l} d h_{j}\left(\vec{h}_{l}\right) \mathbf{J}_{j k} \vec{h}_{k}-2 h_{l} h_{j}\left(v_{l} \mathbf{J}_{j k}\right) \vec{h}_{k}$

$-2 h_{l} h_{j} \mathbf{J}_{j k} \Gamma_{l k}^{i} \vec{h}_{i}-2 H \vec{h}_{0}-h_{0} \vec{H}(\bmod$ vertical $)$ $=-2 h_{i} h_{l} \Gamma_{l j}^{i} \mathbf{J}_{j k} \vec{h}_{k}-2 h_{l} h_{j}\left(v_{l} \mathbf{J}_{j k}\right) \vec{h}_{k}$ $-2 h_{l} h_{j} \mathbf{J}_{j k} \Gamma_{l k}^{i} \vec{h}_{i}-2 H \vec{h}_{0}+h_{0} \vec{H}(\bmod$ vertical $)$ $=-2 h_{l} h_{j}\left(\mathbf{J}_{i k} \Gamma_{l i}^{j}+\mathbf{J}_{j i} \Gamma_{l i}^{k}+v_{l} \mathbf{J}_{j k}\right) \vec{h}_{k}-2 H \vec{h}_{0}+h_{0} \vec{H}(\bmod$ vertical $)$

Since the manifold is Sasakian, we have

$$
\left[\vec{H},\left[\vec{H},\left[\vec{H}, \vec{\alpha}_{0}\right]\right]\right]=-2 H \vec{h}_{0}+h_{0} \vec{H}(\bmod \text { vertical }) .
$$

Proof of Lemma 5.3. Since $\pi_{*} \vec{h}_{j}=v_{j},\left[\vec{h}_{k}, \vec{h}_{i}\right]$ is of the form

$$
\left[\vec{h}_{k}, \vec{h}_{i}\right]=\left(\Gamma_{k i}^{a}-\Gamma_{i k}^{a}\right) \vec{h}_{a}+b_{k i}^{a} \vec{\alpha}_{a}=\mathbf{J}_{k i} \vec{h}_{0}+b_{k i}^{a} \vec{\alpha}_{a}
$$

at $x$. By applying both sides by $d h_{l}$, we obtain

$$
\begin{aligned}
& -b_{k i}^{0}=d h_{0}\left[\vec{h}_{k}, \vec{h}_{i}\right] \\
& =\vec{h}_{k}\left(d h_{0}\left(\vec{h}_{i}\right)\right)-\vec{h}_{i}\left(d h_{0}\left(\vec{h}_{k}\right)\right) \\
& =\vec{h}_{k}\left[\left(\Gamma_{i 0}^{s}-\Gamma_{0 i}^{s}\right) h_{s}\right]-\vec{h}_{i}\left[\left(\Gamma_{k 0}^{s}-\Gamma_{0 k}^{s}\right) h_{s}\right] \\
& =h_{0}\left[\mathbf{J}_{k s} \mathbf{J}_{i s}-\mathbf{J}_{i s} \mathbf{J}_{k s}\right]+h_{s}\left[v_{k}\left(\Gamma_{i 0}^{s}-\Gamma_{0 i}^{s}\right)-v_{i}\left(\Gamma_{k 0}^{s}-\Gamma_{0 k}^{s}\right)\right] \\
& =h_{s}\left[v_{k}\left(\Gamma_{i 0}^{s}-\Gamma_{0 i}^{s}\right)-v_{i}\left(\Gamma_{k 0}^{s}-\Gamma_{0 k}^{s}\right)\right]=h_{s}\left[v_{i} \Gamma_{0 k}^{s}-v_{k} \Gamma_{0 i}^{s}\right]
\end{aligned}
$$

and

$$
\begin{aligned}
& \frac{1}{2} \mathbf{J}_{k i} \mathbf{J}_{l s} h_{s}-b_{k i}^{l}=d h_{l}\left[\vec{h}_{k}, \vec{h}_{i}\right] \\
& =\vec{h}_{k}\left(d h_{l}\left(\vec{h}_{i}\right)\right)-\vec{h}_{i}\left(d h_{l}\left(\vec{h}_{k}\right)\right) \\
& =\vec{h}_{k}\left[\left(\Gamma_{i l}^{a}-\Gamma_{l i}^{a}\right) h_{a}\right]-\vec{h}_{i}\left[\left(\Gamma_{k l}^{a}-\Gamma_{l k}^{a}\right) h_{a}\right] \\
& =-\frac{1}{2} \mathbf{J}_{i l} \mathbf{J}_{k s} h_{s}+\frac{1}{2} \mathbf{J}_{k l} \mathbf{J}_{i s} h_{s}+h_{s}\left[v_{k}\left(\Gamma_{i l}^{s}-\Gamma_{l i}^{s}\right)-v_{i}\left(\Gamma_{k l}^{s}-\Gamma_{l k}^{s}\right)\right]
\end{aligned}
$$


at $x$.

It also follows that

$$
h_{k} b_{k i}^{0}=h_{k} h_{s} v_{k}\left(\Gamma_{0 i}^{s}\right) \text {, }
$$

and

$$
h_{k} b_{k i}^{l}=-h_{s} h_{k}\left[v_{k}\left(\Gamma_{i l}^{s}\right)-v_{k}\left(\Gamma_{l i}^{s}\right)-v_{i}\left(\Gamma_{k l}^{s}\right)\right]
$$

at $x$.

Finally,

$$
\left[\vec{H}, \vec{h}_{i}\right]=h_{k} \mathbf{J}_{k i} \vec{h}_{0}-h_{0} \mathbf{J}_{i k} \vec{h}_{k}+h_{k} b_{k i}^{a} \vec{\alpha}_{a}
$$

\section{REFERENCES}

[1] A.A. Agrachev, P.W.Y. Lee: Bishop and Laplacian comparison theorems on three dimensional contact subriemannian manifolds with symmetry, to appear in J. Geom. Anal., arXiv: 1105.2206 (2011).

[2] A. Agrachev, Y. Sachkov: Control theory from the geometric viewpoint. Encyclopaedia of Mathematical Sciences, 87. Control Theory and Optimization, II. Springer-Verlag, Berlin, 2004.

[3] D.E. Blair: Riemannian geometry of contact and symplectic manifolds. Second edition. Progress in Mathematics, 203. Birkhuser Boston, Inc., Boston, MA, 2010.

[4] D.-C. Chang, I. Markina, A. Vasil'ev: Hopf fibration: geodesics and distances. J. Geom. Phys. 61 (2011), no. 6, 986-1000.

[5] S. Chanillo, P. Yang: Isoperimetric inequalities \& volume comparison theorems on CR manifolds. Ann. Sc. Norm. Super. Pisa Cl. Sci. (5) 8 (2009), no. 2, 279-307.

[6] K. Hughen: The geometry of sub-riemannian three-manifolds, Ph.D. Dissertation 1995.

[7] P.W.Y. Lee: Differential Harnack inequalities for a family of sub-elliptic diffusion equations on Sasakian manifolds, arXiv: 1302.3315 (2013), 27pp.

[8] C.B. Li, I. Zelenko: Differential geometry of curves in Lagrange Grassmannians with given Young diagram. Differential Geom. Appl. 27 (2009), no. 6, 723-742.

[9] C.B. Li, I.Zelenko: Jacobi Equations and Comparison Theorems for Corank 1 sub-Riemannian Structures with Symmetries, Journal of Geometry and Physics 61 (2011) 781-807.

[10] R. Montgomery: A tour of subriemannian geometries, their geodesics and applications. Mathematical Surveys and Monographs, 91. American Mathematical Society, Providence, RI, 2002.

[11] B. O'Neill: Semi-Riemannian geometry. With applications to relativity. Pure and Applied Mathematics, 103. Academic Press, Inc. [Harcourt Brace Jovanovich, Publishers], New York, 1983.

[12] H.L. Royden: Comparison theorems for the matrix Riccati equation. Comm. Pure Appl. Math. 41 (1988), no. 5, 739-746. 
Room 216, Lady Shaw Building, The Chinese University of Hong Kong, Shatin, Hong Kong

E-mail address: wylee@math.cuhk.edu.hk

Department of Mathematics, Tianjin University, Tianjin, 300072, P.R.CHINA

E-mail address: chengboli@tju.edu.cn 